\title{
Perspective
}

PERSPECTIVE Actualité en histoire de l'art

\section{1 | 2019}

\section{Pays nordiques}

\section{La notion de réseau en histoire de l'art : jalons et enjeux actuels}

Network as a Concept in Art History: Milestones and Current Issues

Der Begriff des Netzwerks in der Kunstgeschichte: Etappen und aktuelle Fragen

La nozione di "rete" in storia dell'arte: origine e sviluppi attuali

La noción de red en la historia del arte : referencias y variables actuales

\section{Anne Perrin Khelissa et Émilie Roffidal}

\section{(Q) OpenEdition}

\section{Journals}

Édition électronique

URL : http://journals.openedition.org/perspective/13613

DOI : $10.4000 /$ perspective. 13613

ISSN : 2269-7721

Éditeur

Institut national d'histoire de l'art

Édition imprimée

Date de publication : 30 juin 2019

Pagination : 241-262

ISBN : 978-2-917902-49-3

ISSN : 1777-7852

\section{Référence électronique}

Anne Perrin Khelissa et Émilie Roffidal, « La notion de réseau en histoire de l'art : jalons et enjeux actuels », Perspective [En ligne], 1 | 2019, mis en ligne le 30 décembre 2019, consulté le 01 octobre 2020. URL : http://journals.openedition.org/perspective/13613; DOI : https://doi.org/10.4000/ perspective.13613 


\title{
La notion de réseau en histoire de l'art : jalons et enjeux actuels
}

\author{
Anne Perrin Khelissa et Émilie Roffidal
}

Depuis cinquante ans, la notion de réseau est présentée comme un "nouveau » concept en sciences humaines et sociales. La définition et la variété même des unités lexicales contenant ce terme laissent à deviner l'extrême amplitude de son usage et de son sens : réseaux d'amitié, réseaux familiaux, réseaux socio-professionnels, réseaux intellectuels, réseaux de diffusion, etc. Longtemps attaché au domaine de l'anatomie, de la chirurgie ou de l'histoire naturelle, le terme de " réseau " s'est progressivement imposé, passant de la description de l'activité fluviale et des chemins de fer à celle des relations humaines, et relevant progressivement autant du champ du physique que du conceptuel ${ }^{1}$. Depuis l'essor des outils de communication numériques, qui en ont accru l'action et l'étendue, ce terme est même devenu incontournable. L'étude des réseaux a modifié les approches des chercheurs en sciences humaines et sociales, et l'historien de l'art est incité à prendre part à la dynamique.

Notre réflexion prend racine dans un programme collectif de recherche que nous portons depuis 2016 au sein du laboratoire FRAMESPA (UMR 5136), de l'université Toulouse-JeanJaurès, intitulé Les académies d'art et leurs réseaux dans la France préindustrielle (ACA-RES) ${ }^{2}$. Couvrant un domaine déjà abordé par les historiens et les historiens de l'art - les académies d'art et les écoles de dessin provinciales entre 1740 et 1805 en France ${ }^{3}$-, le projet réinvestit ce corpus sous l'angle des réseaux. Par cette démarche, notre intention est d'ouvrir une lecture jusqu'alors ressérée sur des questions artistiques et pédagogiques, en interrogeant l'institution, eu égard au rôle qu'elle assume dans les carrières des différents acteurs de l'art : du peintre et du sculpteur à l'artisan des manufactures ; de l'homme politique décisionnaire (magistrats des villes ou officiers de l'administration royale) à l'amateur des arts investi dans une culture urbaine. L'enjeu est de comprendre dans quelle mesure les maillages interinstitutionnels et interpersonnels des établissements influent sur l'histoire culturelle et économique du pays. À cette finalité s'est adjointe dès le lancement du programme une exigence méthodologique liée aux nouveaux moyens informatiques et numériques de la recherche. Ces supports de traitement - bases de données textuelles et relationnelles, instruments de visualisation, plateforme d'informations et activation de l'open access, etc.-, loin d'être de simples outils, nous ont amené à redéfinir les cernes et la nature du sujet. 
Ainsi nous souhaitons ici rendre compte de l'amplitude et de la diversité des questionnements soulevés par le programme ACA-RES, dans une perspective large qui interroge la place de notre discipline dans la recherche sur les réseaux. Si cette dernière a été et reste avant tout impulsée par les sociologues, elle a dès le départ considéré le fait artistique comme significatif des processus propres aux réseaux. Le rappel historiographique effectué dans un premier temps a pour vocation de souligner l'intrication inaugurale $\mathrm{du}$ rapport art-société, d'où résulte par la suite l'étude des réseaux proprement dits. Ensuite, notre développement vise à englober les multiples morphologies et implications d'une approche par réseaux, de l'emploi le plus libre, sur un mode métaphorique, à l'utilisation la plus technique, à l'appui de méthodes spécifiques, quantitatives et informatiques. Ainsi s'agira-t-il de considérer l'étude des réseaux, en lien avec mais aussi parfois en marge d'une analyse systématique ou statistique, pour revenir à des questions heuristiques et méthodologiques centrales pour nous : qu'est-ce que l'histoire de l'art prend et apprend de la notion de réseau ? De plus en plus nourrie de l'interdisciplinarité, comment la discipline se place-t-elle vis-à-vis des divers héritages épistémologiques (sociologiques et historiques principalement) et vis-à-vis des perspectives lancées depuis une dizaine d'années par les Humanités numériques?

\section{La rencontre initiale entre art, réseau et sociologie, et ses développements}

Dès 1998, Nathalie Heinich soulignait dans Ce que l'art fait à la sociologie à quel point l'art " incite, plus que tout autre domaine, à opérer des déplacements, qui affectent non seulement la sociologie de l'art, mais l'exercice de la sociologie en général ${ }^{4}$ ". Sans retracer l'historiographie complète de la notion de social network ${ }^{5}$, utilisée pour la première fois dans les années 1950 par l'anthropologue de l'université de Manchester John Barnes (BARNES, [1956] 2013), puis développée et appliquée à partir des années 1970 à l'aide d'informaticiens, rappelons que le monde de l'art a été investi très tôt par les développeurs de ce nouveau mode de compréhension des sociétés humaines. La publication en 1965 des recherches du sociologue Harrison White sur la carrière des peintres en France au XIX ${ }^{e}$ siècle (WHITE, [1965] 1991) en constitue une étape fondamentale. Physicien de formation, White ${ }^{6}$ consacre une année de travail, au sein du Center for Advanced Study in the Behavioral Sciences à Palo Alto, en Californie, à une expérience qui marque la rencontre entre la sociologie et l'histoire de l'art. Le livre qui en est issu est non seulement écrit en collaboration avec son épouse Cynthia A. Johnson, diplômée d'histoire de l'art, mais bénéficie des relectures de deux grands historiens de l'art américain : John Rewald ${ }^{7}$ et Joseph C. Sloane ${ }^{8}$. S'appuyant sur une étude volumétrique, clarifiée et illustrée par une série de tableaux statistiques ${ }^{9}$, les White proposent une analyse partant des " origines de la machine artistique du XIXe siècle " pour arriver jusqu'aux impressionnistes et "leur rôle dans le nouveau système ". Ce livre, Canvases and Careers, même " daté10", possède encore aujourd'hui un intérêt méthodologique ${ }^{11}$. En cherchant à combiner sociologie quantitative et histoire de l'art, les auteurs prennent en compte une multiplicité de facteurs et d'interactions qui éclaire le processus artistique.

Cet ouvrage resta isolé, puisque les publications ultérieures de White et de ses élèves se concentrèrent sur la sociologie économique. Il eut toutefois un fort retentissement, particulièrement en France grâce à Raymonde Moulin. Dès 1966, elle en publiait un compte rendu ${ }^{12}$, relevant l'intérêt de ce type d'analyse pour rendre visible un certain nombre de phénomènes, parmi lesquels la minorité de peintres membres de l'Académie des beaux-arts ayant connu une carrière à succès au cours du XIX siècle (en 1863, seul 1 \% des peintres 
professionnels parisiens sont membres de l'Académie des beaux-arts) ou encore la différence d'attractivité entre arts appliqués et beaux-arts entre la province, où les premiers ont la faveur, et Paris, où ce sont les seconds qui remportent la préférence. L'année suivante, Raymonde Moulin s'en inspire pour détailler le marché de la peinture contemporaine en France (Moulin, 1967). On retrouve également cette " paternité méthodologique " dans un article publié en $1983^{13}$ dans lequel, comme les White, elle fonde son analyse des innovations esthétiques et des transformations de l'organisation professionnelle des mondes artistiques sur une étude de type historique. Comme White, d'ailleurs, elle développe les croisements disciplinaires en entretenant des liens avec des économistes, des philosophes et des historiens de l'art, dont André Chastel ${ }^{14}$.

Les historiens de l'art de l'époque, tout du moins ceux qui développent une approche sociale de l'art, furent sensibles à l'approche développée dans Canvases and Careers. L'ouvrage de White entre ainsi dans les bibliographies de ces derniers : dès 1968, il est présent dans La Vie quotidienne des artistes français au XIX $X^{e}$ siècle de Jacques Lethève ${ }^{15}$. En 1976, il est cité parmi les ouvrages de référence par Enrico Castelnuovo, qui le replace dans le contexte de l'histoire sociale de $\mathrm{l}^{\prime} \mathrm{art}^{16}$. Depuis lors, les travaux de White sont devenus incontournables, ce qui fait écrire à Béatrice Joyeux-Prunel en 2008 : «Quel ouvrage, quel article, quelle thèse, quel mémoire portant sur l'art au XIX ${ }^{\mathrm{e}}$ siècle ne cite pas la fameuse étude de Harrison et Cynthia White ?" (JoyeuX-PRUnel, 2008, p. 22).

Autre jalon sociologique de la notion de réseau appliqué aux mondes de l'art, le vaste panorama qu'Howard Becker dresse en 1982 dans Art Worlds (BECKER, [1982] 1988). Cette publication est le fruit d'un travail initié en 1970 au Center for Advanced Study, là même où White avait conçu son Canvases and Careers. Il doit donc beaucoup aux recherches de White, mais également à celles de Raymonde Moulin, qu'il cite d'ailleurs en introduction et dans son chapitre consacré à la " distribution des œuvres d'art ${ }^{17}$ " (BECKER, [1982] 1988, p. 23 et 27). Considérant que la sociologie de l'art restait un champ sous-développé, Becker s'attache à montrer comment tous les arts (musique, littérature, beaux-arts, etc.) sont « le produit d'une action collective » dont les acteurs partagent des " présupposés communs » et des " conventions " qui leur permettent de " coordonner ces activités efficacement et sans difficultés ${ }^{18}$ " (BECKER, 2013, p. 15-24). Le terme de " monde " vient recouper la notion de réseau de personnes coopérant à faire exister une activité. L'ouvrage rejoint les préoccupations contemporaines de certains historiens de l'art, comme Francis Haskell ou Thomas E. Crow. Francis Haskell, alors professeur d'histoire de l'art à Oxford, envisage les œuvres d'art eu égard au rôle du mécénat, des modes de réception et du phénomène des expositions, que ce soit à Rome, Venise ou Paris ${ }^{19}$. Thomas Crow, quant à lui, dans Painters and Public Life in Eighteenth-Century Paris, détaille les goûts esthétiques des responsables politiques et des élites urbaines, et surtout de la nouvelle classe du public de l'art, critiques et spectateurs qui visitaient le Salon du Louvre ${ }^{20}$. Au-delà des débats passionnés autour des tableaux, des tensions et des oppositions entre les différents groupes en présence, affleure la question des réseaux, qu'ils soient officiels (Académie, Bâtiments du roi) ou parallèles (cercles d'amateurs, expositions non officielles, foires, etc.).

Après Becker, Pierre Bourdieu signale en 1992, dans Les Règles de l'art (BOURDIEU, 1992), l'importance des relations interpersonnelles dans le champ littéraire, en particulier chez Flaubert. Il montre que ce qui le structure est le jeu des interactions entre producteurs, éditeurs, critiques et lecteurs. À ce titre, le concept de " champs " promu par Bourdieu et celui de " mondes " proposé par Becker - que l'on peut cependant chercher à distinguer ${ }^{21}$ - ont l'un et l'autre pour ambition de cerner la dimension collective des pratiques artistiques et culturelles. Cette même année 1992, Raymonde Moulin publie L'Artiste, l'institution et le marché (MouLIN, 1992), où elle développe une approche 
"stratégique " de l'action coordonnée des acteurs en présence. Elle met en évidence les relations qui enjambent la frontière ou l'opposition entre marché et institutions, via les coopérations entre marchands et conservateurs d'art contemporain. Par la prise en compte de cette substance stratégique, l'analyse de réseau dépasse la vision linéaire d'une chaîne d'interaction horizontale à la Becker.

Dans le même temps, Harrison White publie deux ouvrages fondamentaux : Identity and Control (WHITE, 1992) et Careers and Creativity: Social Forces in the Arts (WHITE, 1993). Dans le premier, White prend appui sur ses recherches en sociologie économique et développe une social network analysis, entendue comme une méthode d'analyse structurale permettant de décrire les relations qui unissent les faits, les individus et les structures sociales, de comprendre leurs interactions et de s'interroger sur leur rôle. De concept, le réseau devient méthode d'analyse, avec ses outils, sa méthodologie et son appareil théorique et critique ${ }^{22}$. En France, cette technicité se manifeste dans les travaux d'Alain Degenne et de Michel Grossetti ${ }^{23}$. Leur ouvrage La Vie en réseau, coécrit avec Claire Bidart, marque une étape dans la conceptualisation de ces systèmes et leur possible diffusion auprès des non-spécialistes ${ }^{24}$ (BIDART, DEGENNE, GROSSETTI, 2011).

Dans Careers and Creativity, Harrison White met de côté toute analyse quantitative pour effectuer une synthèse de ses propres recherches sur les pratiques artistiques au regard de celles de Becker, tout en intégrant plusieurs des composantes majeures de sa théorie sur la formation mutuelle des réseaux, des institutions et des organismes. L'ensemble est décrit comme une force agissante construisant les parcours et exaltant l'inventivité25. Depuis lors, la référence aux réseaux est explicite chez nombre de sociologues de la nouvelle génération s'intéressant au domaine artistique, parmi lesquels Guy Bellavance (Bellavance, 2000), Wouter de Nooy (NoOy, 2003), Niels Albertsen et Bülent Diken (Albertsen, Diken, 2004), François Accominotti (ACCOMINOTTI, 2008 ; 2009), Nick Crossley (CROssley, MCANDREW, WIDDOP, 2015) ou encore Martine Azam et Ainhoa de Federico (AZAM, FEDERICO, 2015).

Dans les années 1990, le champ historique est réinvesti de façon conséquente par les chercheurs s'intéressant aux analyses de réseaux, sans toutefois que la rencontre entre sociologie et histoire de l'art soit toujours fluide ${ }^{26}$. En dépit des réserves qui ont pu être exprimées (LEMERCIER, 2005, p. 107), la publication des recherches de John Padgett, en collaboration avec Christopher K. Ansell ${ }^{27}$, sur l'arrivée au pouvoir des Médicis à Florence entre les XIV et $\mathrm{XVI}^{\mathrm{e}}$ siècles constitue une étape fondamentale dans la théorisation de l'organisation des réseaux appliquée aux mondes anciens (PADGETT, ANSELL, 1993). À partir de la collecte de données sur les alliances matrimoniales et les liens économiques entre familles, cette recherche montre la place incontournable des heroes of the new men, au premier rang desquels le riche et puissant Côme de Médicis, qui impulse la domination politique de sa lignée sur l'aire toscane et au-delà. Ces recherches constituent une étape fondamentale dans la théorisation de l'organisation des réseaux appliquée aux mondes anciens : en vingt ans, les auteurs ont développé une base de données relationnelle contenant des informations sur environ 60000 personnes, 10000 mariages, 14000 prêts, 3000 partenariats et entreprises, 40000 enregistrements fiscaux et 12000 élections politiques. Le but était de modéliser la coévolution d'une multitude de réseaux sociaux transversaux dans leur complexité typologique, et ce, à l'échelle de plusieurs générations.

On s'attendrait à ce que ces recherches fondées sur des sources d'archives importantes, et qui portent sur de grands collectionneurs d'art, constituent des points de jonction entre sociologie, histoire et histoire de l'art. Pourtant, il est à noter que la bibliographie de cette publication de 1993, si elle mentionne plusieurs ouvrages d'historiens, ne compte qu'une seule occurrence d'histoire de l'art, particulièrement datée : The Civilization of the Renaissance 
in Italy de Jacob Burckhardt, publié en 1860. Aucune référence dans le texte n'est faite à l'activité de mécène des Médicis, ni aucun lien construit sur la fonction de l'art dans les processus de représentation et de distinction sociales. Une telle absence, si elle n'invalide pas les conclusions tirées, prive cependant d'un éclairage sur les moyens artistiques mis en œuvre pour conquérir et garder le pouvoir. La réciproque est vraie. Les historiens de l'art spécialistes de la Renaissance n'intègrent que très rarement Padgett dans leurs bibliographies.

$\mathrm{Au}$ fil des années, les applications sont toujours plus nombreuses ${ }^{28}$ et de nombreux objets d'étude sont ainsi traités : les élites, les marchands, les communautés religieuses, etc. Les signes de l'attraction exercée par ce concept sont pléthores. Il n'est que de consulter le site internet theses.fr, qui recense l'ensemble des thèses en cours et soutenues en France depuis 1985, pour s'en rendre compte : en 2018, 368 thèses en histoire dont 70 en préparation mentionnent le terme " réseaux " dans leur titre. Autres témoignages : les numéros spéciaux de revues scientifiques dédiés à ce thème ${ }^{29}$; les problématiques soulevées lors des derniers congrès annuels du Comité des travaux historiques et scientifiques $^{30}$; la création, en 2013, du groupe Réseaux et Histoire (RES-HIST) animé par Claire Lemercier et Pierre-Yves Beaurepaire ${ }^{31}$; ou encore la formation du labex Structurations des mondes sociaux (SMS), à Toulouse, qui anime une réflexion interdisciplinaire à travers un atelier méthodologique, Réseaux sociaux Toulouse (ResTo), réunissant sociologues, historiens et historiens de l'art ${ }^{32}$; ou de groupements de recherche (GDR), comme le récent Analyse de réseaux en sciences humaines et sociales (AR-SHS), piloté par des historiens et des sociologues ${ }^{33}$.

\section{Antécédents, habitus et concepts voisins}

La recherche sur les réseaux (comme objet d'étude) et l'analyse de réseau (comme méthode pour les traiter) semblent apporter un degré de précision sans pareil pour définir les types de rapports. Elles aident à mieux envisager ceux qui les engagent et ceux qui les développent, qu'il s'agisse d'individus ou d'entités collectives. Elles tendent à expliciter la qualité du lien et à l'inscrire dans une réalité historique, en tant qu'événement qui porte à conséquence. Au demeurant, les processus décrits, sans nécessairement être nommés, n'étaient-ils pas jadis étudiés par les historiens et les historiens de l'art contextualistes ? Avant que la révolution numérique n'apporte un nouvel outillage aux chercheurs, ceux attachés à la dimension sociale, culturelle et pragmatique de l'art ne pratiquaient-ils pas déjà fréquemment des analyses de réseaux ${ }^{34}$ ?

La monographie, qui est un exercice typique de la discipline de l'histoire de l'art ${ }^{35}$, fait écho, par certains aspects, à l'idée de réseau égocentré émise par les sociologues. Toute monographie demande de s'intéresser à l'état civil d'une personne et de son entourage, et rares sont celles qui ne comportent pas une généalogie. Cette dernière constitue l'état premier d'un graphe de réseau - restreint et axial - permettant de comprendre par quelles ramifications et quels développements se forme une personnalité, naît et existe un individu. L'économiste et historien de l'art John Michael Montias fournit une illustration éloquente du tissu de relations familiales et sociales autour de Vermeer, sans cependant recourir techniquement à la social network analysis (MONTIAS, 1989). Grâce à l'étude minutieuse des protagonistes de son milieu, en particulier son grand-père maternel et sa grand-mère paternelle, il renouvelle la compréhension de l'artiste.

La représentation de la filiation, de la descendance et de l'alliance met en lien, dans le temps et visuellement, des personnes qui ont à voir les unes avec les autres par le sang et le mariage. Documents notariés, testaments, contrats de mariage, actes de baptêmes désignent des parentèles et dénombrent ces complicités. L'historienne de la première Renaissance 
florentine Christiane Klapisch-Zuber détaille l'ancrage des structures familiales, de ces groupes extensifs qui s'appuient sur les liens du sang et sur l'attachement à la corésidence ${ }^{36}$ (KLAPISCH-ZUBER, $1990 ; 2003$ ). De leur côté, les spécialistes des mécanismes curiaux montrent que la domesticité royale fonctionne à l'identique, en version " macro " 37 . En France, les associations se font naturellement, par des filiations directes, ou bien artificiellement, par l'achat d'offices vénaux. Les historiens de l'art qui se consacrent à des analyses de dynasties d'artistes proches du pouvoir politique dressent des constats similaires. Pour les XVII et XVIII ${ }^{\text {e }}$ siècles, songeons parmi tant d'autres aux Hallé ou aux Coypel, ces derniers passant d'une protection à l'autre, d'un poste à responsabilité à un chantier prestigieux, d'une recommandation à l'obtention d'un prix ${ }^{38}$... Dans le domaine de la peinture flamande et hollandaise, le système des réseaux de parentèles est solidement implanté et structure des dynasties entières d'artistes ${ }^{39}$.

Cette réflexion sur les réseaux va de pair avec l'intérêt constant porté à la question de la réussite des carrières, au thème de la célébrité ${ }^{40}$. Lorsqu'un chercheur publie la monographie d'un artiste ou d'un collectionneur, il considère que la personne "mérite ", même modestement, de sortir de l'anonymat. Les traces archivistiques, les témoignages historiques et le corpus des œuvres attestent une certaine importance. Le réseau est alors compris comme un contact qui favorise l'accès à des titres, à des commandes, un marché, une visibilité. Prenons pour exemple la monographie du pastelliste Jean-Baptiste Perronneau, en particulier le chapitre consacré à " Perronneau, voyageur dans l'Europe des Lumières, 1756-178341 ". Dominique d'Arnoult y explique que l'artiste voyage pour asseoir une carrière qui, face à une concurrence intense sur le marché du portrait parisien, oblige l'artiste à aller chercher ailleurs des opportunités. L'auteure emploie l'expression " mobilité de la solidarité " pour définir les deux tours d'Europe engagés par l'artiste, le premier de 1756 à 1764, le suivant de 1767 à 1779. L' « instabilité ", telle que la désignent certains des contemporains de Perronneau, a de fortes conséquences artistiques : devant s'adresser à une clientèle variée, il évite l'écueil d'une production uniforme et codifiée. Elle débouche sur une meilleure réception critique que celle de ses rivaux, dont Quentin de La Tour ${ }^{42}$.

Dès lors que l'on envisage l'artiste dans ses rapports avec ses clients, ses mécènes et ses collectionneurs, le réseau constitue un " capital social " majeur pour le succès d'une carrière (BOURDIEU, 1980 ; COLEMAN, 1988). D'ailleurs l'histoire des collections et du marché de l'art, à côté de l'histoire des artistes et de la création, a privilégié cette problématique, en particulier concernant le rôle des intermédiaires. Le rôle de ces derniers est décisif dans les processus de la commande et pour tout ce qui relève des consommations de l'art (mise en vente, possession, exposition, réception $)^{43}$. Cette dénomination désigne un panel d'acteurs fort différents, de l'expert artistique (qui peut être peintre professionnel, restaurateur, etc.) au courtier, "banquier " qui fait le commerce des tableaux comme des titres de placements. La liste est longue des chercheurs qui ont mis en évidence le rôle des agents, des travaux de Krysztof Pomian ${ }^{44}$ et de Francis Haskell (HASKELL, [1963] 1991) à l'ouvrage de Paolo Cohen sur le marché de la peinture à Rome ${ }^{45}$, en passant par les études d'Olivier Bonfait ${ }^{46}$, de Monica Preti Hamard et Philipe Sénéchal, de Sophie Raux ${ }^{47}$ et de Patrick Michel ${ }^{48}$.

À la vision du réseau comme stratégie de carrière individuelle, il faut ajouter l'exemple du réseau servant un intérêt collectif, au bénéfice d'une communauté. Ainsi en est-il du

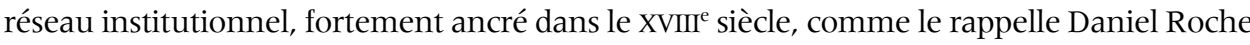
dans l'avant-propos ${ }^{49}$ de l'ouvrage dirigé par Pierre-Yves Beaurepaire sur les réseaux de correspondances (BEAUREPAIRE, 2002) ou bien dans son livre Humeurs vagabondes ${ }^{50}$. Mais y a-t-il contradiction, opposition, entre la formation d'un réseau qui sert des intérêts personnels et de celui qui sert des intérêts institutionnels ? À quel moment et dans quelle 
mesure les deux se rencontrent-ils ? Les discussions engagées lors des premières journées d'étude du programme ACA-RES, en décembre 2016, ont permis de mettre en relief la combinaison de ces facteurs ${ }^{51}$. Les projets de fondation d'écoles de dessin mettent en avant l'utilité sociale d'un enseignement en reprenant les arguments habituels de l'occupation de la jeunesse, du développement industriel, de l'amélioration du goût ${ }^{52}$. Ils répondent en même temps au souhait de quelques hommes d'exercer leur métier ou leur pouvoir dans la cité : les artistes multiplient les démarches (auprès de la direction des Bâtiments, de la municipalité, de protecteurs privés) pour accéder à un poste de professeur ou de directeur d'école ; les membres fondateurs (notables locaux, politiques, parlementaires, intendants) luttent entre eux pour répartir et revendiquer leur rôle.

Quoi qu'il en soit, l'académie d'art régionale formalise et officialise l'existence d'un réseau, autant qu'elle en crée un nouveau, par la voie des conditions d'accès et de l'enseignement. Les modes d'entrée dans l'institution s'attachent à différencier les membres entre eux, sur l'argument des compétences et des qualités des récipiendaires, ainsi qu'en fonction de

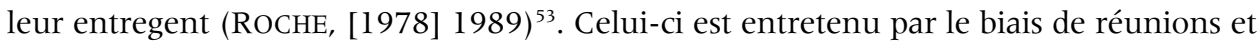
d'assemblées, de devoirs et d'obligations vis-à-vis du corps. Dans le contexte d'un corps académique, d'une communauté qui requiert de l'aspirant son admission par un processus d'assentiment collectif, la notion de réseau peut paraître contradictoire. Elle contrevient en effet à l'image idéale que veut se donner l'institution. Elle se place en porte-à-faux d'une rhétorique dont l'argumentation se base sur la sélection des stricts talents comme reconnaissance et récompense du génie ${ }^{54}$. Le relationnel interfère dans l'élimination ou, au contraire, la célébration des personnalités, si bien que l'institution donne lieu à des discours $\mathrm{d}^{\prime}$ opposition $^{55}$. À une vision idéale de l'artiste candidat, ancrée dans une tradition classique forgée depuis la Renaissance, on oppose l'idée d'une structure disciplinaire, contraire à la liberté, où la compétition est instrumentalisée sous l'habillage de l' " émulation " positive. Les critiques portées contre l'Académie royale de Paris, durcies sous la Révolution française, révèlent ce paradoxe ${ }^{56}$. En aval, l'académie d'art, au cœur d'un réseau, produit un bien immatériel : la formation artistique, l'amélioration du goût des peintres, des sculpteurs aussi bien que des amateurs, et la sociabilité artistique qui permet de faire du dessin un terrain de rencontres et d'échanges entre personnes. Dans ce contexte, et par effet de ricochets, le réseau s'étend au maillage économique.

Tout en étant anachronique pour la période moderne, la notion de réseau a l'avantage de rester neutre et plus objective que des phénomènes qui lui sont proches. Elle peut participer d'une histoire de la sociabilité, mais elle ne lui est pas nécessairement conditionnée ${ }^{57}$. Il en est de même pour le concept voisin de mondanité. Le " réseau " ne renvoie pas derechef à la société "policée " des Lumières. Il n'entre donc pas nécessairement dans la filiation d'une histoire culturelle ${ }^{58}$. L'historien Antoine Lilti, dans Le Monde des salons, ne met pas de côté l'aspect concret des réseaux, en envisageant toutes les pratiques de cette forme de sociabilité ${ }^{59}$. Il s'en sert du reste pour démontrer que les salons ont un sens social et politique décisif : il s'agit pour les élites aristocratiques de renégocier une domination hors du cadre de la cour, dans l'espace urbain, et de faire de l'expression artistique et intellectuelle un geste qui ne soit pas uniquement celui du prince. La place prise par les amateurs au XVIII ${ }^{\mathrm{e}}$ siècle comme nouvelles figures des mondes de l'art est exemplaire de ce type de phénomènes où s'entrecroisent débats inédits et nouvelles activités artistiques, où basculent les rapports entre les différents hommes de goût. Charlotte Guichard l'a montré dans son livre dédié à ces figures ${ }^{60}$.

Le réseau porte en lui une grande puissance de malléabilité sociale. Il fait évoluer les cadres socio-culturels d'une société, d'une communauté. Il devient alors plus directement connecté à la notion de changement, d'évolution, de mutation. La franc-maçonnerie est 
à ce titre représentative ${ }^{61}$. Elle constitue l'un des premiers cercles utopiques dissidents, en ce qu'elle voit se rencontrer, outre des hommes de lettres, des philosophes et des aristocrates, comme cela s'observait dans les salons mondains, des hommes de métiers et de classes inférieures. Au XVIII siècle, son déploiement dans l'espace européen, s'il n'est pas rendu public, est équivalent à l'étendue de l'Église catholique. La franc-maçonnerie, cercle sélectif et en partie secret, offre à certains la possibilité de rencontrer des groupes sociaux vers lesquels ils n'auraient pu se tourner publiquement à la " surface sociale " (BIDART, Degenne, GrossetTi, 2011, p. 53). Dans le champ artistique, l'efficacité du réseau se mesure également à cette capacité de dépassement d'un état " premier ». Le gain tiré est celui de la chose apportée (la commande par exemple) et celui du miroitement positif produit par le contact. Citons le cas des petits maîtres qui parviennent, via leur réseau, soit à gravir la hiérarchie des chantiers, soit à bénéficier de l'aura de ceux, artistes et ateliers plus prestigieux, qu'ils côtoient ${ }^{62}$.

\section{Outils numériques et histoire de l'art}

La recherche sur les réseaux en histoire de l'art s'est emparée depuis une dizaine d'années de l'outil numérique, en particulier des bases de données, en lien avec le développement des projets ANR, émanations du Programme d'investissements d'avenir. La méthodologie pensée pour la constitution de notre base de données ACA-RES a nécessité d'être interrogée à trois moments clefs : en amont, dans la considération des sources documentaires ; au cœur du traitement de l'information, quand il s'est agi d'établir une classification et une indexation normalisée ; en aval, enfin, lorsqu'il faut présenter et valoriser les résultats obtenus ${ }^{63}$.

La question des sources est particulièrement en jeu. Notre corpus, composé des fonds d'archives relatifs aux académies d'art et écoles de dessin ${ }^{64}$, peut-il se réduire à des données normées et/ou quantitatives? N'est-ce pas passer outre le caractère fragmentaire, partiel et fragile du matériel des sciences humaines, qu'il s'agisse de sources d'archives pour l'historien ou l'historien de l'art, mais également des données collectées par les sociologues? Les sources ne sont ni neutres, ni objectives, et elles sont toujours le résultat conjoint de stratégies discursives et des aléas de la conservation ${ }^{65}$. Les documents manuscrits eux-mêmes ne sont pas standardisés et peuvent correspondre ou pas à des rubriques proposées dans la base de données. Le processus demande de délimiter un corpus cohérent de sources : soit il s'agit de sources de seconde main qui, de fait, ne tiennent que partiellement compte des avancées de la recherche, de la découverte de nouveaux documents et de révisions critiques ; soit il s'agit de sources de première main et, dans ce cas, la difficulté réside dans le traitement et l'équilibrage de données souvent disparates. Les problématiques liées aux sources impactent en conséquence directement les axes de la recherche. Comment ne pas comprendre alors la place prédominante des supports textuels pour travailler sur les réseaux en sciences humaines et sociales ${ }^{66}$ ? Les recherches ciblées ${ }^{67}$ ou plus ambitieuses sur les correspondances font florès : le programme Mapping the Republic of Letters, abrité par l'université de Stanford ${ }^{68}$, le projet Van Gogh Letters, porté par le Van Gogh Museum $\mathrm{d}^{\prime}$ Amsterdam ${ }^{69}$, notamment, illustrent cette aspiration à manipuler un matériau « fiable » . Les collections déjà existantes de correspondances fournissent des séries d'informations "brutes "- dates, destinataires et expéditeurs - qui permettent une collecte systématique. Toujours dans cette volonté de collecter des données uniformisées à grande échelle, croisant une démarche extensive de global history, Maximilian Schich s'est appuyé sur les dates et les lieux de naissance et de décès de 100000 individus entre l'an 600 et 2012 pour dresser « a network framework of cultural history ${ }^{70}$. 
Le mode de traitement du matériau collecté soulève d'autres interrogations. Les systèmes informatiques et les logiciels utilisés, eux aussi le fait de l'homme, sont faillibles et peuvent introduire des biais par erreur de codage. Les bases de données soutiennent autant qu'elles mettent en danger le travail de l'historien de l'art. Elles l'invitent en tout cas à interroger ses instruments de traitement des données. Les questions terminologiques également sont sensibles pour convenir d'un vocabulaire commun : l'utilité d'unifier et de convenir d'un vocabulaire est indispensable à la création d'une base de données. L'histoire de l'art n'est pas novice en la matière, stimulée en cela par le service de l'Inventaire général, de la publication, en 1972, de l'Architecture par Jean-Marie Pérouse de Montclos ${ }^{71}$, détaillant le vocabulaire et l'analyse scientifique des éléments d'architecture, à celle, en 1999, du Thésaurus des objets religieux ${ }^{72}$ détaillant les termes en trois langues, suivi par le Thésaurus des objets mobiliers ${ }^{73}$. L'historien de l'art peut ainsi s'appuyer sur une tradition ancienne de programmes d'indexation de termes contrôlés, normalisés et hiérarchisés. Dans le cas d'ACA-RES, un travail réflexif sur ce qu'est un " décorateur ", un " collectionneur " ou un " amateur " a dû précéder le recensement des activités des individus dans la base. Mais quels sont les critères pour en décider?

Cette question en apparence simple est rapidement confrontée à la réalité des sources, plus ambiguës qu'il n'y paraît, d'autant que doivent être prises en compte leurs dates d'exercice, structurantes dans le contexte d'une base de données relationnelle, où l'identité des acteurs et la nature de leurs liens sont en jeu. Il ne s'agit pas de produire une liste d' " amateurs " ou de "décorateurs ", mais bien de comprendre des dynamiques de connexions et d'interdépendances. La question terminologique devient celle de la caractérisation de ces liens. S'il semble assez évident de différencier un lien "professionnel » d'un lien " amical ", la confrontation aux sources montre que le passage de l'un à l'autre est fréquent et parfois confus. De plus, l'enjeu est de décrire et de qualifier l'action de chaque individu dans l'ensemble du réseau. À ce titre, la réflexion des sociologues sur la spécification et la qualité des liens constitue une réelle ressource. Relevons deux exemples : la notion de " centralité de proximité " (closeness centrality) définie par Linton Freeman $^{74}$, qui calcule le degré d'autonomie ou d'indépendance des acteurs (FREEMAN, 2004) - "plus un acteur est éloigné des autres acteurs, plus il sera autonome dans ses choix d'actions "-, et celle de l'intensité du lien, promue par Mark Granovetter (GRANOVETTER, 1973), qui permet de caractériser les liens « forts » et les liens « faibles ». Ces deux notions sont éclairantes pour analyser, notamment, les carrières d'artistes et les stratégies de progression sociale de commanditaires.

D'un point de vue opératoire, l'enjeu est finalement l'interrogation de la base de données et la justesse des résultats produits. Certains problèmes peuvent émerger : la corrélation entre traitement de l'information et possibilités d'interrogation, la question de la dynamique des liens, de leur diachronie et de leur temporalité, la prise en compte de l'absence des liens, etc. Autre interrogation, celle de la valeur ajoutée du graphe. Selon la théorie des graphes, "la figuration graphique dépasse[rait] la simple représentation pour autoriser un traitement mathématique générateur de connaissances nouvelles " (MERCKLÉ, 2003-2004, p. 12-14). En théorie, les graphes permettent de mettre en évidence des phénomènes que la source textuelle ne donne pas d'elle-même, comme la densité et la connexité d'un réseau. Les questions sont pourtant nombreuses autour de leur justesse : par exemple, amoindrissent-ils le caractère égotique au profit du collectif (HASSLER, 2015) ? N'écrasent-ils pas des événements qui ont un impact fort sur l'inconscient collectif? Ne consentent-ils pas surtout à produire un schéma de synthèse qui valide des intuitions (BIDART et al., 2010, p. 11)? 
Plusieurs voix émettent des constats nuancés dans la communauté des historiens de l'art. Béatrice Joyeux-Prunel, en 2008, s'interroge : " pourquoi tant de bases de données, de répertoires, de catalogues en ligne, mais si peu d'utilisations de ces gisements de données ${ }^{75}$ ? " (JOYEUX-PRUNEL, 2008, p. 4). Les porteurs du programme Arachné - Méthode critique de l'histoire de la tapisserie : préceptes, circulation de modèles, transferts de savoir-faire - expliquent leurs réserves pour la mise en ligne de leur base de données relationnelles, consacrée aux tapissiers et à leur entourage, actifs en France entre les XIV e et XXI ${ }^{\mathrm{e}}$ siècles. La base, "opérationnelle et pérennisée ", nécessite en effet une interface pour être mise à disposition des chercheurs (KARSALLAH et al., 2017). Les 15000 entrées, les 4900 acteurs et les 1000 documents traités supposent, pour être interrogés, une connaissance du domaine de la tapisserie, de ses techniques et de ses questionnements propres, et surtout une formation préalable pour en comprendre le fonctionnement, les enjeux et les limites.

Les chercheurs travaillent donc à trouver des solutions, car l'apport scientifique et pédagogique est avéré. Concernant la visualisation des résultats obtenus par les bases de données relationnelles, l'étude menée par Jean-Pierre Dedieu sur les individus qui ont appartenu aux trois principales académies royales espagnoles au XVIII ${ }^{\mathrm{e}}$ siècle - Académie royale de la langue, Académie royale d'histoire et Académie royale des beaux-arts, situées à Madrid - a permis de produire des schémas montrant l'articulation des points de contact (DEDIEU, CHAPARRO, 2013). Il apparaît que les membres affiliés conjointement à ces trois institutions sont peu nombreux, en comparaison de la myriade d'individus qui gravitent autour d'un même cercle. Le projet de grande ampleur ECARTICO, quant à lui, sur le réseau des ateliers néerlandais à l'époque moderne, parvient à rendre accessible des phénomènes complexes en mobilisant le cognitif et le ludique ${ }^{76}$. Par ailleurs, le projet SymoGIH, portant sur la modalisation et la gestion de l'information historique, s'est donné pour mission de stocker des données historiques structurées, des données spatiales ainsi que des textes encodés provenant de programmes de recherche différents ${ }^{77}$. L'idée est de se libérer des schémas liés à des problématiques particulières, de décomposer les connaissances en " unités atomisées " afin qu'elles soient réinterrogées et éventuellement réutilisées par d'autres chercheurs.

\section{Le réseau comme clef d'analyse utile à notre discipline}

Dans ses modalités tant métaphoriques que techniques, le concept de réseau est susceptible de mettre en évidence des phénomènes artistiques particulièrement signifiants et permet notamment de retracer la progression des carrières. Il prend un sens particulier dans le cas d'artistes étrangers qui doivent s'établir sur un territoire et trouver les moyens de se faire une place. La contribution récente de Gaëtane Maës sur Jean-Baptiste Descamps est tout à fait éclairante à ce sujet ${ }^{78}$. Comme l'auteure l'écrit dans son introduction, l'analyse de la carrière de " cet étranger en France qui parvient à se hisser au sein de l'élite de son pays d'adoption ${ }^{79}$ " comporte un élément atypique. Dans la partie de l'ouvrage consacrée à la "Stratégie de distinction [de Descamps] ${ }^{80}$ ", Gaëtane Maës explique que sa tactique a consisté à cultiver son statut d'étranger (né à Dunkerque et formé à Anvers) pour se différencier dans un milieu concurrentiel et non à se fondre dans la masse en adoptant ses codes. La notion d'écart et de distance parcourue la conduit à la conclusion suivante : "Descamps n'a jamais occupé de place centrale ou décisionnaire dans la politique des arts, mais sa position en retrait de modeste peintre installé en province ne l'a pas empêché de se placer au cœur de questions d'actualité, 
pour lesquelles son expertise s'est rapidement révélée indispensable ${ }^{81}$. " Le " réseau ", quand il est saisi dans ses diverses facettes, à entrées multiples, permet de dépasser l'idée d'une réciprocité binaire.

Mobilité sociale et mobilité géographique sont donc deux grands mouvements qui parcourent les réseaux. La question des voyages, des circulations, est centrale et recoupe la problématique des transferts artistiques et culturels, elle aussi vive ces dernières années ${ }^{82}$. "Le changement de perspective que constitue le passage d'une logique des grands ensembles et des cohérences spatiales à une logique des circulations individuelles, des déplacements identifiés et des réseaux qui les expliquent, transforme profondément la géographie de l'art traditionnelle ${ }^{83}$ ". Lorsqu'elle est soutenue, administrée voire instrumentalisée par l'État, cette mobilité, ce "vagabondage ", pour reprendre le terme qu'emploie Alain Bonnet dans son ouvrage consacré à L'Artiste itinérant ${ }^{84}$, transforme autant la production artistique que l'image de l'artiste. Les deuxièmes journées d'étude du programme ACA-RES ont mis l'accent sur cet aspect du problème ${ }^{85}$. S'interroger sur les modes de déplacement des ouvres autant que des hommes conduit à envisager les phénomènes d'appropriation, de transposition et d'adaptation. D'autres programmes de recherche en ont fait auparavant leur axe, comme celui porté par France Nerlich sur les artistes allemands en France et les artistes français en Allemagne ${ }^{86}$. La force des réseaux se mesure souvent à son pouvoir d'outrepasser les frontières. Le réseau agit hors d'un territoire donné, hors d'une configuration préalable, et crée un résultat inattendu.

La caractéristique du réseau, contrairement au simple contact, est donc de produire un effet, voire un développement. White l'avait d'ailleurs théorisé avec le concept de " commutation" (switching), marquant l'efficience des réseaux qui ne sont pas de simples " tuyaux " servant la circulation des ressources (BIDART et al., 2010, p. 4). Si ce développement peut être un bien immatériel, il intéresse davantage l'historien de l'art lorsqu'il s'incarne dans l'œuvre. Bénédicte Gady, dans l'introduction de son ouvrage L'Ascension de Charles Le Brun - qui fait de l'essor d'une carrière son questionnement principal -, énonce les intentions de son étude : mettre au jour « les mécanismes d'une ascension exceptionnelle et la construction d'une carrière, là aussi en faisant la part de l'héritage, des réseaux, du talent, de l'habileté, de l'audace voire de la chance ? ", tout en maintenant "une curiosité pour les seconds rôles et les intermédiaires ". Il est nécessaire de " comprendre des réseaux de relations que les individus acceptent pour une part et transforment pour l'autre " (GADY, 2010, p. 5) et de veiller à ce que les réseaux soient utilisés comme une clef d'analyse parmi d'autres. Le réseau ne suffit pas à lui seul à expliquer le succès, le seul talent non plus. En outre, le réseau ne se réduit ni à une causalité, ni à un système. L'auteure explique que ce travail d'analyse des liens sociaux confrontés à la production artistique de Le Brun jusqu'aux années 1661-1662 ne définit pas une grille d'analyse interchangeable. Si jusqu'au retour d'Italie de Le Brun, en 1646, l'étude conjointe des relations et des œuvres « fait ressortir une correspondance, sinon systématique, du moins très étroite, entre réseaux, commandes et styles des œuvres " (GADY, 2010, p. 8), après cette date, du fait que la demande provient en priorité du sommet de l'État, la réalité devient plus complexe. En l'absence de sources documentant les liens sociaux, comme dans le cas de l'hôtel Lambert, Bénédicte Gady fait le choix de ne pas appliquer la méthode élaborée pour d'autres chantiers. Ainsi le réseau et, surtout, ses liens avec les ouvres ne déterminent pas un état de fait systématique, une lecture imparable.

Un autre cas offert par l'actualité de la recherche réintroduit l'œuvre d'art au cœur d'une réflexion sur les réseaux. Hannah Williams consacre une partie importante de son ouvrage sur les portraits académiques aux processus d'accession au rang académique et au rôle qu'y jouent les arguments d'amitiés et de parentèle, d'affinités et d'allégeance 
(WILLIAMS, 2015). Dans ce fonctionnement, le portrait est un objet qui matérialise un rapport, un réseau. La réalisation de l'effigie de ses confrères, en particulier pour les morceaux d'agrément et de réception, marque une affiliation, un attachement à une personne. Le portrait est un moyen de faire sa cour ou d'exposer à une assemblée son entregent. Il est parfois partie prenante de la naissance du lien ${ }^{87}$. Comme le souligne l'auteure, il entre dans le processus de don et de contre-don qu'avait décrit Marcel Mauss $^{88}$. Marquée par les apports de l'anthropologie, sensible à la notion d'agency diffusée par Alfred Gell ${ }^{89}$, l'auteure conçoit le tableau comme preuve mais aussi agent du lien, démarche qui fait naître des interprétations subtiles. Retenons l'exemple du double autoportrait de Jean-Baptiste de Champaigne et Nicolas de Plattemontagne, qui semble être construit tel un rébus désignant, peut-être sur le mode de l'invitation au jeu, un type de relation singulier ${ }^{90}$. Ces deux artistes quasiment du même âge érigent par la peinture un monument (au sens premier d'objet mémoriel) à leur amitié. Ils scellent ensemble leur relation en mêlant leur pâte et leur propre image dans l'œuvre. L'auteure suggère " que ces artistes ont trouvé un moyen d'effacer la couture unissant leur main dans un acte suprême de réciprocité. Si la composition est lue comme un récit de la fabrication de l'objet, alors la peinture elle-même fournit tous les indices nécessaires pour comprendre sa paternité ${ }^{91}$. " Pour Champaigne, c'est aussi une manière de renouer avec une tradition flamande dont il est issu, celle de ces tableaux collaboratifs que peignaient notamment les amis Pierre Paul Rubens et Jan Brueghel l'Ancien" ${ }^{92}$.

L'autre objet-relai, à la fois paramètre et élément du réseau, pourrait être le livre. On retrouve ici l'intérêt pour les sources textuelles qui constituent un matériau de premier plan pour l'étude des réseaux. Roger Chartier et les spécialistes de l'édition de l'Encyclopédie de Diderot et d'Alembert ont montré combien cette entreprise était un chantier d'une infinie complexité, où des intérêts multiples ont été soudés ${ }^{93}$. Des entreprises éditoriales comme l'Histoire naturelle de Buffon ${ }^{94}$ ou les recueils d'estampes artistiques ${ }^{95}$ ont tout autant fait se rencontrer des compétences et des aspirations diverses, scientifiques et artistiques, intellectuelles et commerciales, pour un produit qui les sublimait toutes. Le colloque organisé par Yves Chevrefils-Desbiolles et Rossella Froissart sur les revues d'art au XX $\mathrm{XX}^{\mathrm{e}}$ siècle, "Formes, stratégies et réseaux " (CHEVREFILS-DESBIOLLES, FroIsSARTPEZONE, 2011), a bien mis en évidence ces connexions. Plusieurs revues consacrées aux arts sont envisagées par leurs créateurs comme des éléments constitutifs d'une stratégie collective d'autopromotion. Elles sont un moyen de diffuser de nouveaux concepts esthétiques, autant de tribunes politiques pour faire entendre des convictions ou des oppositions, et elles favorisent en même temps la création. Elles entrent dans un rapport de force revigoré vis-à-vis de l'institution et modifient le champ artistique ${ }^{96}$. Par la suite, l'ouvrage imprimé, quand il est agencé, organisé, mis " en voisinage " avec d'autres au sein des bibliothèques, quand il fait l'objet d'une manipulation et d'une discussion active, instaure des " chaînes d'associations " qui créent ensemble une culture. Tel était le projet d'Aby Warburg pour sa Kulturwissenschaftliche Bibliothek, que certains considèrent comme son œuvre majeure ${ }^{97}$. À l'aide d'une classification normalisée moins complexe et subjective, les bibliothèques proposant une consultation en accès libre des ouvrages favorisent les connexions intellectuelles, individuelles et collectives. Elles ménagent des espaces de surprise, admettent l'irruption du " chaos ", stimulant par l'expérience vécue la recherche heuristique.

Le phénomène des réseaux, raisonné en concept et éclairé par des outils d'analyse numériques, lie dans des cadres spatio-temporels élargis des faits et des éléments qui paraissaient au premier abord éloignés. Il crée des passerelles et met en synergie l'action d'individus, dessinant un maillage d'interconnexions toujours plus extensives. Cette complexité semble 
approcher au plus près d'une réalité historique et correspond à l'idée familière que les historiens de l'art se font de la richesse de leur domaine d'étude. Toutefois la méthodologie, encore perfectible, constitue un point de frottement entre ambitions et épreuve de terrain. On est en droit de se demander si les désirs et les fantasmes projetés sur les bases de données ne surestiment pas leur réelle potentialité. Au-delà de cette interrogation, il apparaît que $l^{\prime}$ histoire de l'art a toute sa place dans cette phase d'expérimentation ${ }^{98}$. Alors que les bases de données iconographiques qui ont vu le jour dans les musées (Joconde) et dans les services de l'architecture et du patrimoine (Mérimée, Palissy) ont mis des décennies avant de pouvoir être réellement exploitées, puis intégrées dans des bases encore plus vastes, les bases de données relationnelles devraient pouvoir dépasser ces difficultés. Déjà le regroupement effectué par l'INHA sur AGORHA de l'ensemble des bases de données résultant de ses programmes de recherche montre que l'interconnexion et l'actualisation des informations sont possibles.

Par ailleurs, l'apport du numérique ne se réduit pas à l'utilisation des bases de données relationnelles et la mise en réseaux des connaissances passe par des voies multiples. L'ensemble des ressources documentaires que sont susceptibles de véhiculer des plateformes numériques comme Nakalona (mise en œuvre par la très grande infrastructure de recherche Huma-Num), dans la continuité de la politique de numérisation des fonds culturels initiée dans les années 1990, constituent des supports de recherche incomparables. Les nouvelles formes de la diffusion des connaissances, comme les carnets de recherche Hypothèses, sont aussi aptes à faire avancer la réflexion. Par leur forme collaborative et évolutive, ils fonctionnent comme un work in progress en ligne. Largement accessibles aux spécialistes et au grand public, ils autorisent plus facilement l'intégration de points de vue divergents et de méthodes concurrentes au sein d'un même espace de discussion. Le numérique invite également davantage au dialogue intergénérationnel ${ }^{99}$.

De fait, ces différents outils modifient notre façon d'exercer notre métier d'historien de l'art. Les attendus sociétaux qui investissent le monde de la recherche se renforcent. Ils requièrent sans doute une vigilance accrue de la part des chercheurs. Tout en écartant une lecture déterministe des réseaux, il faut cependant leur reconnaître un certain nombre de vertus. Ils constituent tout d'abord un apport sémantique indiscutable. Ils permettent ensuite d'appréhender autrement des sujets déjà balisés, de rouvrir des débats, de confirmer des approches et d'en renouveler certaines. Par exemple, ils participent de l'enrichissement de l'histoire globale ${ }^{100}$. En se situant du côté d'une histoire des conditions de la production artistique, et non du côté de l'étude de la production elle-même, ils complètent l'approche stylistique et iconographique. Dans le cas des académies d'art et des écoles de dessin de province au XVIII ${ }^{e}$ siècle, des perspectives s'ouvrent. Si on les considère uniquement sous l'angle de la pédagogie artistique, leurs bilans semblent contrastés : elles ne répondent pas, le plus souvent, aux objectifs premiers énoncés dans leurs règlements ; leur instabilité structurelle (remises en cause, fermetures et réouvertures dues au changement de gouvernements et aux problèmes financiers) a sans cesse menacé leur fonctionnement ; la réputation des quelques " grands " noms sortis de leur rang reste aléatoire et toujours dépendante d'une historiographie artistique valorisant Paris ${ }^{101}$. Dès lors que ces institutions sont envisagées dans leurs rapports entre elles et avec d'autres cercles, pays ou domaines, dès lors qu'elles sont vues comme des espaces de négociations et d'échanges entre des interlocuteurs d'horizons et de statuts divers, elles redonnent une certaine texture aux territoires. Entre spécificités des milieux et impulsion européenne, micro- et macro-histoire, elles font émerger la partie cachée des processus qui lient art et société. 


\section{Anne Perrin Khelissa}

Maître de conférences en histoire de l'art moderne à l'université Toulouse - Jean Jaurès, coresponsable de l'équipe de recherche «CréationProduction " du laboratoire FRAMESPA-UMR 5136, Anne Perrin Khelissa est spécialiste de l'art du XVIII ${ }^{\mathrm{e}}$ siècle. Elle s'intéresse aux arts décoratifs pris dans leurs rapports avec les autres arts, à la culture matérielle et à l'histoire du luxe, aux discours et à l'enseignement artistiques. Elle a écrit notamment sur l'ameublement intérieur, les circulations entre la France et I'Italie, la porcelaine et les manufactures royales, les conférences de I'Académie royale de peinture et de sculpture et celles de l'Académie de Lyon.

\section{Émilie Roffidal}

Chargée de recherche au CNRS, Émilie Roffidal est rattachée au laboratoire FRAMESPA-UMR 5136 où elle développe des recherches sur les liens interacadémiques en Europe méridionale. Elle s'intéresse aux circulations artistiques - avec un intérêt particulier pour les sculpteurs-, aux réseaux socioculturels, aux interactions des artistes avec les mondes productifs et à la culture matérielle des objets sur un large XVIII ${ }^{\mathrm{e}}$ siècle. Ses publications les plus récentes sont consacrées à l'Académie de peinture et de sculpture de Marseille, étude de cas porteuse pour interroger ces différentes notions.

\section{NOTES}

Nous souhaitons remercier Michel Grossetti, Gaëtane Maës et Sébastien Poublanc pour leur relecture et leurs conseils. Nos remerciements vont également aux collaborateurs d'ACA-RES et aux intervenants des journées d'étude, qui, au fil des échanges et des discussions, ont enrichi notre réflexion. Enfin nous tenons à remercier l'équipe et le comité de la revue Perspective pour la qualité de leur expertise et de leur suivi éditorial.

1. Ce n'est que dans la huitième édition du Dictionnaire de l'Académie française (1932-1935) qu'il est écrit : "Réseau s'emploie aussi figurément. Exemple : Un réseau d'intrigues".

2. On peut consulter la page Hypothèses du programme ACA-RES - Les académies d'art et leurs réseaux dans la France préindustrielle [en ligne, URL : acares.hypotheses. org], où est offert en accès libre l'ensemble de nos données et de nos résultats de recherche. Ainsi le lecteur pourra y trouver autant la numérisation indexée des sources d'archives, que l'état de l'art et les publications issues de nos activités, des actualités, etc.

3. Par " académies d'art ", nous entendons des institutions académiques qui comportaient le domaine artistique parmi leurs attributions, sachant que certaines académies étaient seulement consacrées aux beaux-arts, comme à Toulouse et à Marseille, alors que d'autres associaient les arts aux sciences et aux belles-lettres, comme à Lyon ou à Bordeaux. L'intégration, ou non, de l'architecture, dépendait, elle aussi, des multiples situations locales. Les écoles de dessin, qui font partie du corpus des établissements étudiés, dépendaient généralement de l'académie quand elle existait dans la ville. Nous reviendrons plus loin sur les études fondamentales sur le sujet. Voir par ailleurs le point historiographique livré sur la page Hypothèses du programme.

4. Nathalie Heinich, Ce que l'art fait à la sociologie, Paris, Les Éditions de Minuit, 1998.

5. En effet, ce travail historiographique a déjà été réalisé, notamment par MERCKLÉ, 2004 ; FREEMAN, 2004 ; AZAM, FEDERICO, 2015.

6. White fait partie de l'école de Chicago. Outre Chicago, il s'est formé et a enseigné dans les universités de Princeton et d'Harvard. Pour une introduction à certains aspects de ses recherches, voir Michel Grossetti et Frédéric Godart, "Harrison White : des réseaux sociaux à une théorie structurale de l'action ", dans SociologieS, Découvertes/Redécouvertes, Harrison White [mis en ligne le 17 octobre 2007, URL : sociologies.revues.org/233, consulté le 10 octobre 2017] ; AZARIAN, 2005.

7. John Rewald avait publié en 1946 un très remar qué History of Impressionism, New York / Greenwich, Museum of Modern Art / New York Graphic Society, 1946. Élève d'Erwin Panofsky à Hambourg puis d'Henri Focillon à la Sorbonne, il est professeur à Princeton et à Chicago avant d'enseigner à New York, où il travaille également pour le Museum of Modern Art. Spécialiste de Paul Cézanne, il œuvre pour que soit transformé en musée l'atelier du peintre à Aix-en-Provence. Il est d'ailleurs enterré près de la tombe de ce dernier. 
8. Joseph Curtis Sloane publie en 1951 French Painting Between the Past and the Present: Artists, Critics, and Traditions from 1848 to 1870, Princeton, Princeton University Press, 1951. Professeur et directeur du département d'histoire de l'art de l'université de Caroline du Nord, Chapel Hill, il fut également directeur du North Carolina Museum of Art.

9. À titre d'exemple : « Répartition en pourcentages du nombre de peintures vendues, par nationalité et par catégorie, sur quatre périodes, lors des ventes aux enchères parisiennes des principales collections »; « Prix des peintures d'histoire, par nationalité », faisant une sorte de carottage sur les périodes : 1737-1756 ; 17571776 ; 1817-1837 et 1838-1857; « Caractéristiques des carrières des peintres français du XIXe siècle " proposant un pourcentage des peintres issus de l'École des beaux-arts, ceux nés à Paris, ceux qui reçurent des commandes officielles, etc.

10. Ce constat est déjà fait par Jean-Paul Bouillon dans la préface de la réédition de 1991.

11. Il n'est pas exclu cependant de critiquer la méthode, qui ne prend pas appui sur des sources primaires mais sur des publications, comme les ouvrages de Ludovic Vitet, de Clara Harrison Stranahan et de Lionello Venturi.

12. Raymonde Moulin, "White H. C, White C. A., Canvases and Careers: Institutional Change in the French Painting World ", dans Revue française de sociologie, $\mathrm{n}^{\circ} 7-3,1966$, p. 400-402.

13. Raymonde Moulin, "De l'artisan au professionnel, l'artiste ", dans Sociologie du travail, n ${ }^{\circ} 4,1983$, p. 388-403.

14. Sur le sujet, on peut écouter la conférence donnée par Pierre-Michel Menger le 16 mars 2017 à la Bibliothèque nationale de France [en ligne, URL : www. bnf.fr/fr/evenements_et_culture/anx_conferences_2017/ a.c_170316_petite_bibliotheque_sciences_sociales.html, consulté le 12 décembre 2017]. Plus généralement sur les liens entre sociologues et économistes, voir PierreMichel Menger, « Les artistes en quantités. Ce que sociologues et économistes s'apprennent sur le travail et les professions artistiques ", dans Revue d'économie politique, $n^{\circ} 120 / 1,2010$, p. 205-236. Sur les liens entre la recherche en économie et en histoire de l'art, on peut se reporter aux travaux de John Mickael Montias, à l'université de Yale, sur le prix des œuvres d'art aux PaysBas, notamment MONTIAS, 1982.

15. Jacques Lethève, La Vie quotidienne des artistes français au XIX siècle, Paris, Hachette, 1968.

16. Enrico Castelnuovo, "L'histoire sociale de l'art, un bilan provisoire ", dans Actes de la recherche en sciences sociales, no 6, décembre 1976, p. 63-75; idem, Arte, industria, rivoluzioni, temi di storia sociale dell'arte, Turin, G. Einaudi, 1985.

17. Il est d'ailleurs à noter qu'entre son article « Art of collective actions » dans l'American Sociological Review en 1974 et le livre Art Worlds en 1982, Becker a visiblement lu Raymonde Moulin.

18. Howard S. Becker, Propos sur l'art, Alain Pessin, Pascale Ancel, Alain Blanc et al. (éd.), Jean Kempf, JeanMarie Fournier, Vincent Michelot et al. (trad. fra.), Paris / Montréal, L'Harmattan, 1999, p. 99.
19. Francis Haskell, Rediscoveries in Art: Some Aspects of Taste, Fashion, and Collecting in England and France, Londres, Phaidon, 1976.

20. Thomas E. Crow, Painters and Public Life in EighteenthCentury Paris, New Haven / Londres, Yale University Press, 1985. Cet ouvrage a été traduit et réédité à plusieurs reprises.

21. Dans un entretien donné à Alain Pessin en 2006, Howard Becker cherche à distinguer l'un de l'autre (BECKER, PESSIN, 2006).

22. Pour comprendre la différence entre l'école structuraliste et une « sociologie conventionnelle » plus qualitative, voir EVE, 2002.

23. Voir FERRAND, FEDERICO, 2013.

24. Notons que Michel Grossetti a collaboré à la version française de Identity and Control (Harrison C. White, Identité et contrôle. Une théorie de l'émergence des formations sociales, Frédéric Godart et Michel Grossetti (trad. fra.), Paris, Éditions de l'EHESS, 2011). Depuis les années 1980, l'étude des réseaux en France était le fait de spécialistes qui diffusaient leurs recherches sur le site internet « Réseaux sociaux » de l'Association française de sociologie ou dans la revue Réseaux, communication, technologie, société, éditée par l'université ParisEst et créée en 1982. Une publication récente permet de faire le bilan de cet apport de "l'école française » de sociologie : PENALVA-ICHER, ELOIRE, 2017. On peut également consulter : BAUGUITTE, 2016.

25. Voir en particulier dans WHITE, 1993, I'introduction intitulée "Art World and Agency » (p. 8-11) et le chapitre "Creativity and Agency " (p. 95-118).

26. À ce titre, on peut citer les recherches menées et encadrées par Maurizio Gribaudi, qui se fondent sur une série d'études de relations égocentrées pour comprendre des dynamiques sociales plus globales : GRIBAUDI, 1998.

27. Padgett est professeur de sciences politiques à l'université de Chicago. Voir aussi PADGETT, 2001 ; 2010.

28. Si les titres n'affirment pas, dans un premier temps, une dimension réticulaire, le phénomène est cependant bien au cœur d'une série de travaux au cours des années 1990, par exemple : Claire Dolan, Le Notaire, la famille et la ville. Aix-en-Provence à la fin $d u X V l^{e}$ siècle, Toulouse, Presses universitaires du Mirail, 1998 ; PaulAndré Rosenthal, Les Sentiers invisibles. Espace, famille et migrations dans la France du XIXe siècle, Paris, Éditions de I'EHESS, 1999.

29. LemerCier, 2005 ; idem, "Conclusion » du dossier "Réseaux », dans Hypothèses, 2010, p. 293-300 ; BERTRAND, GUZZI-HEEB, LEMERCIER, 2011a ; 2011b ; SENMARTIN, BERTRAND, 2015.

30. On mentionnera notamment celui de Reims en 2015 sur « Réseaux et société »; voir la liste complète [en ligne, URL : cths.fr/co/liste.php (consulté le 18 novembre 2017)].

31. Voir le site internet du groupe RES-HIST : reshist. hypotheses.org, consulté le 20 novembre 2017.

32. Voir le site internet du labex SMS : sms.univ-tlse2.fr/ accueil-sms/le-labex-sms, consulté le 20 janvier 2018.

33. Parmi ceux-ci, on retrouve Pierre-Yves de Beaurepaire, Laurent Beauguitte, Claire Lemercier et Serge Lhomme. 
Site internet du GDR AR-SHS : arshs.hypotheses.org, consulté le 20 janvier 2018.

34. Pour une anthologie de textes mettant en avant ces optiques, Johanne Lamoureux, Neil McWilliam, Constance Moréteau (dir.), Histoires sociales de l'art. Une anthologie critique, Paris / Dijon, INHA / Les presses du réel, 2016.

35. Position qu'affirme, entre autres, Pierre Rosenberg dans l'introduction au numéro consacré à cette question de la revue Perspective, n ${ }^{\circ}$ 4, 2006 ("La monographie, un outil indispensable», p. 497-498). L'exercice de la monographie a pu être mis en cause par des approches plus globalisantes (Cultural et Visual Studies).

36. Sur ces questions, on peut également mentionner les travaux d'Anna Bellavitis, dont Famille, genre, transmission à Venise au XVI siècle, Rome, Publications de I'École française de Rome, 2008 ; ou idem, Identité, mariage, mobilité sociale. Citoyennes et citoyens à Venise au XVI siècle, Rome, Publications de l'École française de Rome, 2013, en particulier la troisième partie intitulée "Alliances, mobilité sociale et mémoire familiale ».

37. Voir récemment l'axe de recherche porté par Mathieu Da Vinha au Centre de recherche du château de Versailles sur les "Réseaux et sociabilité de cour ", qui met en avant le rôle de la transmission des offices dans l'ascension de certaines familles et la hiérarchisation des maisons royales.

38. Nicole Willk-Brocard, Une dynastie. Les Hallé : Daniel (1614-1675), Claude-Guy (1652-1736), Noël (17111781), Paris, Arthéna, 1995 ; sur les Coypel, voir Nicole Garnier-Pelle, Antoine Coypel, Paris, galerie de Bayser, 1989 ; Thierry Lefrançois, Charles Coypel, peintre du roi : 1694-1752, Paris, Arthéna, 1994 ; Jérôme Delaplanche, Noël-Nicolas Coypel, Paris, Arthéna, 2004.

39. Voir notamment BROSENS, KELCHTERMANS, VAN DER STIGHELEN, 2012.

40. Sur ce thème, pour un point historique et historiographique, voir Antoine Lilti, Figures publiques. L'invention de la célébrité (1750-1850), Paris, Fayard, 2014. Parmi les figures étudiées sont privilégiés les hommes et femmes publics, gens de la scène théâtrale (comédiens), mondaine (philosophes tels Voltaire et Rousseau) et politique. Plusieurs études collectives de monographies d'artistes accordaient une grande importance à cette question, en particulier Guilhem Scherf (dir.), Augustin Pajou et ses contemporains, actes de colloque (Paris, 1997), Paris, La Documentation française, 1999 ; DÉCULTOT, ESPAGNE, MARTIN, 2009.

41. Dominique d'Arnoult, Jean-Baptiste Perronneau. Un portraitiste dans l'Europe des Lumières, Paris, Arthéna, 2014, p. 131 et suiv.

42. Bien que d'autres commentateurs « imputeront au voyage l'oubli dans lequel l'artiste est tombé à Paris à la fin de sa carrière ", Arnoult, 2014, cité n. 41, p. 160.

43. Pour un point historiographique sur le rôle des intermédiaires, à partir du compte rendu de quatre ouvrages dont ceux de Charlotte Guichard et Patrick Michel ici cités, voir Noémie Étienne, "Intermédiaires et mondes de l'art à Paris au XVIII' siècle : approches et méthodes comparées ", dans Perspective, nº 1, 2011, p. 489-493. Du même auteur, sur une des figures d'intermédiaires, à savoir le restaurateur, voir La Restauration des peintures à Paris (1750-1815). Pratiques et discours sur la matérialité des œuvres d'art, Rennes, PUR, 2012. Là encore, nous constatons qu'une césure chronologique est instaurée en débutant par la seconde moitié du siècle.

44. Krysztof Pomian, Collectionneurs, amateurs et curieux : Paris, Venise, XVI $-X V I I I^{e}$ siècles (1978), Paris, Gallimard, 1987.

45. Paolo Cohen, Il mercato dei quadri a Roma nel Diciottesimo secolo. La domanda, l'offerta e la circolazione delle opere in un grande centro artistico europeo, Florence, Leo Olschiki, 2010.

46. Olivier Bonfait, Les Tableaux et les Pinceaux. La naissance de l'école bolonaise (1680-1780), Rome, Publications de l'École française de Rome, 2000.

47. Monica Preti-Hamard, Philippe Sénéchal (dir.), Collections et marché de l'art en France, 1789-1848, actes du colloque (Paris, Institut national d'histoire de l'art, 2003), Rennes, Presses universitaires de Rennes, 2005. Sophie Raux (dir.), Collectionner dans les Flandres et la France du Nord au XVIII siècle, actes de colloque (Lille, 2003), Villeneuve-d'Ascq, Presses de l'université Charles-de-Gaulle-Lille 3, 2005. Sophie Raux a par ailleurs dirigé le projet financé par l'Agence nationale de la recherche (ANR) Art Markets in Europe, 1300-1800: Emergence, Development, Networks, qui déboucha sur le projet Mapping Art Markets in Europe, 15001800 , dont une présentation, avec une visualisation des résultats, est consultable sur le site web du projet [en ligne, URL : www.artmarkets.eu/presentation, consulté le 2 février 2018].

48. Patrick Michel, Le Commerce du tableau à Paris dans la seconde moitié du XVIII' siècle, Villeneuve d'Ascq, Presses universitaires du Septentrion, 2007.

49. Daniel Roche, "Avant-propos », dans BEAUREPAIRE, 2002, p. 7-24.

50. Daniel Roche, Humeurs vagabondes. De la circulation des hommes et de l'utilité des voyages, Paris, Fayard, 2003. Consulter en particulier le chapitre « Les jeux de la mobilité : hiérarchisation et institutionnalisation des mobilités savantes", p. 707-728. Le rôle du réseau académique, institutionnel, est défini comme I'« armature de la République des Lettres».

51. Voir le compte rendu détaillé de cette rencontre : "Actes des journées d'étude 1 ", ACA-RES, [en ligne, URL : acares.hypotheses.org/les-papiers-daca-res/actesdes-journees-detude, consulté le 25 novembre 2017].

52. Le cas de Rouen peut être cité, avec le discours de Descamps où il est question de la fondation de l'école de Bachelier l'année précédente. Voir Aude Gobet, "Entre normes pédagogiques et utilité sociale. "Sur I'utilité des établissemens des écoles gratuites de dessein de Jean-Baptiste Descamps (1767)" » dans Thomas Gaehtgens et al. (dir.), L'Art et les normes sociales au $X V I I I^{e}$ siècle, Paris, Éditions de la Maison des sciences de I'homme, 2001, p. 313-331.

53. Agnès Lahalle, Les Écoles de dessin au XVIII' siècle : entre arts libéraux et arts mécaniques, Rennes, PUR, 2006 ; Reed Benhamou, Regulating the Académie: Art, Rules and Power in Ancien Régime France, Oxford, Voltaire Foundation, 2009. 
54. Nathalie Heinich, Du peintre à l'artiste. Artisans et académiciens à l'âge classique, Paris, Les Éditions de Minuit, 1993.

55. Depuis la seconde moitié du XVIII' siècle et surtout au XIX ${ }^{e}$ siècle (depuis Jacques-Louis David), I'Académie est au contraire dénoncée comme un pouvoir réfractaire à la modernité et à l'originalité, que ce soit dans son recrutement, ses positions ou son fonctionnement; Dominique Poulot, "L'Académie saisie par la modernité ? Sur l'espace public de la peinture en France au XVIII siècle ", dans Revue d'histoire moderne et contemporaine, $\mathrm{n}^{\circ}$ 37/1, janv.-mars 1990, p. 108-127.

56. Christian Michel, L'Académie royale de peinture et de sculpture : la naissance de l'École française, Genève, Droz, 2012, p. 154-168. On peut également citer le projet soutenu par le Fonds national suisse de la recherche scientifique intitulé L'art et la démocratie : la naissance d'une question. Les réflexions sur la place et le statut des arts du dessin (juillet 1789 - août 1792).

57. Bien qu'elle soit " équivoque ", la notion de sociabilité est ainsi préférée, contre celle de " réseau », dans I'introduction de : Jessica L. Fripp et al. (dir), Artistes, savants et amateurs. Art et sociabilité au XVIII siècle (1715-1815), actes de colloque (Paris, 2011), Paris, Mare \& Martin, 2016, p. 13-26. Se référer aussi à la thèse de troisième cycle fondatrice : Maurice Agulhon, «La sociabilité méridionale. Confréries et associations dans la vie collective en Provence orientale à la fin du XVIII" siècle" dans Publications des Annales de la Faculté des lettres, Aix-en-Provence, série Travaux et Mémoires, nº 36, 1966, rééd. en 1968.

58. En dehors de Paris, sur les sociabilités provinciales, voir Daniel Roche, Les Républicains des Lettres. Gens de culture et Lumières au XVIII siècle, Paris, Fayard, 1988. Voir également Katia Béguin, Olivier Dautresme (dir.), La Ville et l'esprit de société, actes de la journée d'études (Tours, 2002), Tours, Presses universitaires François-Rabelais, 2004, dans lequel on trouve des articles de Pierre-Yves Beaurepaire (« Franc-maçonnerie, espace public et vie de société », p. 33-53), de Charlotte Guichard (" Hors l'Académie, les amateurs et les expositions artistiques publiques à Paris : le Musée de Pahin de la Blancherie [1777-1788] », p. 55-72) et d'Antoine Lilti (« Espace urbain, espace mondain : Paris et la sociabilité mondaine au XVIII ${ }^{\mathrm{e}}$ siècle », p. 111-127).

59. Antoine Lilti, Le Monde des salons. Sociabilité et mondanité à Paris au XVIII siècle, Paris, Fayard, 2005.

60. Charlotte Guichard, Les Amateurs d'art à Paris au XVIII siècle, Seyssel, Champ Vallon, 2008.

61. Renvoyons surtout aux très nombreux travaux de Pierre-Yves Beaurepaire, parmi lesquels Pierre-Yves Beaurepaire, La République universelle des francsmaçons. De Newton à Metternich, Rennes, Éditions Ouest France, 1999 ; idem, L'Europe des francs-maçons : XVIII'-XXI siècle, Paris, Belin, 2002 ; ainsi qu'à la récente exposition de la Bibliothèque nationale de France et son catalogue : Sylvie Bourel, Pierre Mollier, Laurent Portes (dir.), La Franc-maçonnerie, cat. exp. (Paris, BnF, 2016), Paris, BnF, 2016

62. Récemment, et directement rattaché à une histoire des réseaux, voir BRESC-BAUTIER, 2017.
63. Le manuel d'utilisation de notre base de données relationnelles est accessible sur la page Hypothèses ACARES, à la rubrique «Autres ressources/Base de données ».

64. Les sources sont issues des Archives nationales et, pour chaque ville, des archives départementales, municipales et fonds anciens des bibliothèques municipales ou des académies encore existantes. Sur le traitement des archives, voir la rubrique «Corpus d'archives NAKALONA » sur notre page Hypothèses.

65. Camille Bloomfield, "Du document à l'archive : I'historien de la littérature face à ses sources", dans Littérature, no $166,2012-2$, p. 69-83 [en ligne, URL : www.cairn.info/revue-litterature-2012-2-page-69.htm, consulté le 15 janvier 2018].

66. Un rapport sur les Humanités numériques commandé par I'Institut français soulignait d'ailleurs à quel point la " linguistique computationnelle » était présente dès les premiers âges des Humanités numériques : stylométrie, lexicométrie, etc. Voir DACOS, MOUNIER, 2015

67. Nous citerons ici deux études, l'une en histoire et l'autre en histoire de l'art : Françoise Deroy-Pineau, Paul Bernard, "Projet mystique et stratégie réticulaire de mobilisation des ressources : Marie Guyart, mystique et stratège ", dans Pierre-Yves Beaurepaire, Dominique Taurisson (dir.), Les Ego-documents à I'heure de l'électronique. Nouvelles approches des espaces relationnels, actes de colloque (Montpellier, 2002), Montpellier, Publications de l'université Paul-Valéry, 2003, p. 399420 ; Mathilde Arnoux, Thomas W. Gaehtgens, Anne Tempelaere-Panzani (dir.), Correspondance entre Henri Fantin-Latour et Otto Scholderer, 1858-1902, Paris, Éditions de la Maison des sciences de l'homme (Passages/Passagen, 24), 2011.

68. Mapping the Republic of Letters [en ligne, URL : republicofletters.stanford.edu, consulté le 3 mai 2017].

69. Site internet du programme Van Gogh Letters [en ligne, URL : vangoghletters.org/vg, consulté le 23 novembre 2017]. Sur l'historique et la structuration de ce programme, on peut se reporter à Marianne Jakobi, «L'édition électronique des lettres d'artistes : le cas Van Gogh », dans Perspective, $\mathrm{n}^{\circ}$ 2, 2011 [mis en ligne le 30 juin 2013, URL : journals.openedition.org/perspective/814, consulté le 4 janvier 2018].

70. Historien de l'art de l'université de Dallas, Maximilian Schich a publié en 2014 : Maximilian Schich et al., "A Network Framework of Cultural History ", dans Science, 345/6196, 2014, p. 558-562. Une vidéo intitulée "Charting Culture » (2014) présente les résultats du programme [en ligne, URL : www.youtube.com/ watch? $v=4$ glhRkCcD4U, consulté le 18 avril 2019]. Un autre programme peut être cité, celui d'Hannah Williams intitule " Artists in Paris. Mapping the $18^{\text {th }}$-Century Art World » (2015-2018) [en ligne, URL : www.artistsinparis.org]. Il localise à Paris 450 artistes membres de I'Académie royale de peinture et de sculpture entre 1675 et 1793

71. Jean-Marie Pérouse de Montclos, Architecture. Vocabulaire. Inventaire général des monuments et des richesses artistiques de la France, Francis Salet et Sylvain Stym-Popper (éd.), Paris, Imprimerie nationale, 1972. 
72. Joël Perrin, Sandra Vasco Rocca (dir.), Thésaurus des objets religieux. Meubles, objets, vêtements et instruments de musique du culte catholique romain, Paris, Éditions du Patrimoine, 1999.

73. Hélène Verdier (dir.), Thésaurus des objets mobiliers, Paris, Éditions du Patrimoine (Inventaire général des monuments et des richesses artistiques de la France), 2001.

74. Linton Freeman est également fondateur de la revue Social Networks en 1978.

75. Une publication rend particulièrement compte de ces difficultés : Franziska Heimburger et Émilien Ruiz, « Faire de l'histoire à l'ère numérique : retours d'expériences" ", dans Revue d'histoire moderne et contemporaine, $n^{\circ}$ 58/4 bis, 2011-5, p. 70-89.

76. La base ECARTICO, Linking cultural industries in the early modern Low Countries, ca. 1475-ca. 1725, est portée par l'université d'Amsterdam. Nous remercions Stéphanie Trouvé de nous avoir signalé l'existence de ce projet de recherche.

77. Voir le site internet du projet SymoGIH [en ligne, URL : symogih.org, consulté le 12 janvier 2018]. Pour en comprendre la méthode et les enjeux, voir BERETTA, VERNUS, 2012 ; BERETTA, 2017 ; BERETTA, LETRICOT, 2017.

78. Gaëtane Maës, De l'expertise artistique à la vulgarisation au siècle des Lumières : Jean-Baptiste Descamps (1715-1791) et la peinture flamande, hollandaise et allemande, Turnhout, Brepols, 2016.

79. Maës, 2016, cité n. 78, p. 13.

80. Maës, 2016, cité n. 78, p. 140-142.

81. Ibidem.

82. Jan Blanc, Gaëtane Maës, "Introduction. Pour une étude dynamique des échanges artistiques ", dans Les Échanges artistiques entre les anciens Pays-Bas et la France, 1482-1814, acte de colloque (Lille, 2008), Turnhout, Brepols, 2010, p. 7-14.

83. Jacques Dubois, Jean-Marie Guillouët, Benoît Van den Bossche, "Le "déplacement" comme problème : les transferts artistiques à l'époque gothique ", dans idem (dir.), Diffuser l'art gothique. Les transferts artistiques en Occident du XII au XVI siècles, Paris, Picard, 2014, p. 9-34.

84. Alain Bonnet, L'Artiste itinérant. Le Prix du Salon et les bourses du voyage distribuées par l'État français (1874-1914), Paris, Mare \& Martin, 2016.

85. Les actes sont en cours de publication.

86. Voir notamment NERLICH, 2009 ; ainsi que l'ouvrage issu de sa thèse de doctorat, France Nerlich, La Peinture française en Allemagne, 1815-1870, Paris, Éditions de la Maison des sciences de l'homme, 2010. L'auteure y étudie l'action que la présence d'œuvres françaises a pu exercer sur le territoire allemand par leur circulation, leur présence dans les collections et les expositions, l'intégration des artistes français au sein des institutions et des sociétés savantes et la transformation du regard porté sur l'art dans un contexte de questionnement identitaire national. Voir également France Nerlich, Alain Bonnet (dir.), Apprendre à peindre. Les ateliers privés à Paris au XIXe siècle, actes de colloque (Tours, 2011), Tours, Presses universitaires François-Rabelais, 2013 et le projet ANR/DFKG ArtTransform : Formations artistiques transnationales entre la France et l'Allemagne, 1789-1870, en collaboration avec Bénédicte Savoy, présenté dans NERLICH, SAVOY, 2016.

87. WILliams, 2015, chapitre V : "Reciprocal Acts: Portraits of Friendship », p. 208-250.

88. Auquel fait référence Hannah Williams; rappelons le titre de l'ouvrage fondateur de Mauss, qui connut de nombreuses éditions et traductions, Marcel Mauss, Essai sur le don. Forme et raison de l'échange dans les sociétés archaïques (1925), Paris, PUF, 2012.

89. En particulier son ouvrage pionnier : Alfred Gell L'Art et ses agents. Une théorie anthropologique, Sophie Renaut et Olivier Renaut (trad. fra.), Dijon, Les presses du réel, 2009 [éd. orig. : Art and Agency: An Anthropological Theory, Oxford, Clarendon Press, 1998].

90. Le tableau, de 1654, est conservé à Rotterdam, au Museum Boijmans Van Beuningen. Le passage qui lui est consacré dans l'ouvrage d'Hannah Williams (WILLIAMS, 2015) est p. 215-221.

91. WILLIAMS, 2015, p. 218 : «that these artists found a way to efface the joining seam of their hands in the ultimate act of reciprocation. If the scene is read as a narrative of the object's making, then the painting itself provides all the clues necessary for understanding its authorship. »

\section{WOOllett, VAN SUCHTELEN, 2006.}

93. Roger Chartier, Henri-Jean Martin (dir.), Histoire de l'édition française, Paris, Fayard, 1989-1991. La bibliographie sur l'Encyclopédie serait trop longue à introduire ici ; renvoyons au site internet de l'édition numérique collaborative et critique (ENCREE) [en ligne, URL : enccre.academie-sciences.fr/encyclopedie, consulté le 12 janvier 2018].

94. On pourra consulter le site internet porté par le CNRS et conçu sous la direction de Thierry Hoquet, Buffon et l'histoire naturelle : l'édition en ligne [en ligne, URL : www.buffon.cnrs.fr, consulté le 20 octobre 2017].

95. Cordélia Hattori, Estelle Leutrat, Véronique Meyer (dir.), À l'origine du livre d'art : les recueils d'estampes comme entreprise éditoriale en Europe, XVI'-XVIII' siècles, actes de colloque (Paris, 2006), Milan, Silvana Editoriale, 2010.

96. Citons également les travaux de Juliette Lavie sur plusieurs revues du XX $x^{e}$ siècle, notamment La Revue de la photographie, publiée périodiquement dans les années 1930, ou bien les "sœurs » franco-allemandes des revues d'art graphiques Gebrauchsgraphik et Arts et Métiers graphiques. Voir Juliette Lavie, "La revue, un outil de transmission des savoirs d'ateliers? L'exemple d'Emmanuel Sougez dans la revue PHOTOCiné-GRAPHIE (1933-1936) », dans Alain Bonnet, Juliette Lavie, Julie Noirot et al. (dir.), Art et transmission. L'atelier du XIXe au XXI siècle, actes de la journée d'études (Paris, 2009), Rennes, PUR, 2014, p. 175-188; eadem, "Gebrauchsgraphik, un modèle esthétique et intellectuel pour la revue des Arts et Métiers graphiques (1927-1937) ? ", dans Anne-Rachel Hermetet, Nathalie Prince (dir.), Pour une cartographie européenne des revues littéraires (1909-1939). Relations, réseaux, carrefours, actes de colloque (Angers, 2009), s. d., p. 143-152 
[en ligne, URL : www.vox-poetica.com/sflgc/a/IMG/ pdf/.pdf, consulté le 15 janvier 2018].

97. Maud Hagelstein, « Mémoire et Denkraum. Réflexions épistémologiques sur la Kulturwissenschaftliche Bibliothek Warburg ", dans Conserveries mémorielles, $\mathrm{n}^{\circ}$ 5, 2008 [en ligne, URL : journals.openedition.org/ $\mathrm{cm} / 104$, consulté le 4 février 2018].

98. C'est ce que propose de faire l'unité mixte InVisu (CNRS/INHA), née en 2008, pour l'expérimentation en histoire de l'art des nouvelles technologies de l'information et de la communication.

99. Nous renvoyons sur ces différents aspects à Marin Dacos, "Manifeste des Digital humanities ", THATCamp Paris, 26 mars 2011 [en ligne, URL : tcp.hypotheses. org/318, consulté le 18 avril 2019].

100. Citons seulement, parmi un grand nombre de publications, RIELLO, 2007 ; ainsi que Natacha Coquery, Caroline Varlet, «Introduction », dans Histoire urbaine, $\mathrm{n}^{\circ}$ 4, 2001-2, p. 5-7 [en ligne, URL : www.cairn.info/ revue-histoire-urbaine-2001-2-page-5.htm, consulté le 23 juillet 2016].

101. Alain Bonnet arrive à des constats semblables quand il étudie la réforme de l'École des beaux-arts en 1863, soulevant aussi l'idée - séculaire - que le génie artistique ne peut se réduire à un " apprentissage » ; Alain Bonnet, L'Enseignement des arts au XIX siècle. La réforme de l'École des Beaux-Arts de 1863 et la fin du modèle académique, Rennes, PUR, 2006.

\section{BIBLIOGRAPHIE SÉLECTIVE SUR LES RÉSEAUX}

- ACCOMINOTTI, 2008 : François Accominotti, «Marché et hiérarchie. La structure sociale des décisions de production dans un marché culturel ", dans Art et mesure, numéro thématique de Histoire \& Mesure, ${ }^{\circ}$ 23/2, 2008, p. $177-218$.

- ACCOMINOTTI, 2009 : François Accominotti, «Creativity from Interaction: Artistic Movements and the Creativity Careers of Modern Painters ", dans Poetics, $n^{\circ}$ 37/3, juin 2009, p. 267-294.

- ALBERTSEN, DIKEN, 2004 : Niels Albertsen, Bülent Diken, "Artworks' Networks Field, System or Mediators?", dans Theory, Culture \& Society, no 21/3, 2004, p. 35-58. - AZAM, FEDERICO, 2015 : Martine Azam, Ainhoa de Federico, "Sociologie de l'art et analyse des réseaux sociaux ", dans Sociologie de l'Art, $\mathrm{n}^{\text {os }}$ 25-26, 2015-1, p. 13-36.

- AZARIAN, 2005 : Gholam Reza Azarian, The General Sociology of Harrison C. White: Chaos and Order in Networks, Basingstoke / New York, Palgrave Macmillan, 2005.

- BARNES, (1956) 2013 : John A. Barnes, «Classes sociales et réseaux dans une île de Norvège ", Jean Grange (trad. fra.), dans Réseaux, no 182, 2013-6, p. 209-237 [en ligne, URL : www.cairn.info/revue-reseaux-2013-6-page-209. htm, consulté le 30 janvier 2018].

- BAUGUITTE, 2016 : Laurent Bauguitte, "L'analyse de réseaux en sciences sociales et en histoire : Vocabulaire, principes et limites", dans Rosemonde Letricoat et al. (dir.), Le Réseau. Usages d'une notion polysémique en sciences humaines et sociales?, Louvain, Presses universitaires de Louvain, 2016.

- BeAurepaIRE, 2002 : Pierre-Yves Beaurepaire (dir.), La Plume et la Toile. Pouvoirs et réseaux de correspondance dans l'Europe des Lumières, actes de colloque (Arras, 2000), Arras, Presses de l'université d'Artois, 2002.

- Beaurepaire, HäSEler, MCKenNA, 2006 : PierreYves Beaurepaire, Jen Häseler, Antony McKenna (dir.), Réseaux de correspondance à l'âge classique (XVI $X V I I I^{e}$ siècles), actes de colloque (Lyon/Los Angeles, 2003), Saint-Étienne, Publications de I'université de Saint-Étienne, 2006.

- BECKER, 1974 : Howard S. Becker, «Art of Collective Actions ", dans American Sociological Review, 1974, p. 767-776.

- BECKER, (1982) 1988 : Howard S. Becker, Les Mondes de l'art, Pierre-Michel Menger (présentation), Jeanne Bouniort (trad. fra.), Paris, Flammarion, 1988 [éd. orig. : Art Worlds, Berkeley, University of California Press, 1982]. - BECKER, 2013 : Howard S. Becker, " Avant-propos. Inventer chemin faisant : comment j'ai écrit Les mondes de l'art ", dans Pierre-Jean Benghozi et Thomas Paris (dir.), Howard Becker et les mondes de l'art, actes de colloque (Cerisy, 2010), Palaiseau, Éditions de l'École Polytechnique, 2013, p. 15-24.

- BECKER, PESSIN, 2006 : Howard S. Becker, Alain Pessin, "Dialogue sur les notions de Monde et de Champ ", dans Sociologie de l'Art, n' ${ }^{\circ}$, 2006-1, p. 163-180.

- BellavanCE, 2000 : Guy Bellavance (dir.), Monde et réseaux de l'art. Diffusion, migration et cosmopolitisme en art contemporain, Montréal, Liber, 2000. 
- BERETTA, 2017 : Francesco Beretta, «L'interopérabilité des données historiques et la question du modèle : I'ontologie du projet SyMoGIH », dans Brigitte Juanals et Jean-Luc Minel (dir.), Enjeux numériques pour les médiations scientifiques et culturelles du passé, actes de la rencontre (LabEx « Les passés dans le présent »), Nanterre, Presses universitaires de Paris Nanterre, 2017. - BERETTA, LeTRICOT, 2017 : Francesco Beretta, Rosemonde Letricot, "Le portail XML du projet symogih. org : un projet d'édition numérique collaborative de sources et d'informations historiques ", dans Gérald Kembellec et Évelyne Broudoux (dir.), Écrilecture augmentée dans les communautés scientifiques. Humanités numériques et construction des savoirs, Londres, ISTE Éditions, 2017, p. 125-143.

- BeretTA, VernuS, 2012 : Francesco Beretta, Pierre Vernus, "Le projet SyMoGIH et la modélisation de I'information : une opération scientifique au service de I'histoire ", dans Les Carnets du LARHRA, 2012, p. 81-107.

- Bertrand, GuZZI-HeEB, LeMERCIER, 2011a : Michel Bertrand, Sandro Guzzi-Heeb, Claire Lemercier (dir.), Analyse de réseaux en histoire : outils, approches, problèmes, numéro thématique de Redes. Revista Hispana para el Análisis de Redes Sociales, n 21, décembre 2011. - BertRAND, GuZZI-HEEB, LeMERCIER, 2011b : Michel Bertrand, Sandro Guzzi-Heeb, Claire Lemercier (dir.), "Introduction : où en est l'analyse de réseaux en histoire ? " / "Introducción al análisis de redes e historia: herramientas, aproximaciones, problemas », dans BERTRAND, GUZZI-HEEB, LEMERCIER, 2011a, p. 1-12 et p. 13-23. - BIDART et al., 2010 : Claire Bidart et al., "Réseaux sociaux : une "French Touch" ? Retour sur l'école thématique CNRS "Réseaux sociaux : enjeux, méthodes, perspectives" (Cargèse [Corse], 15-20 septembre 2008) ", dans Bulletin de Méthodologie Sociologique, $\mathrm{n}^{\circ}$ 106/1, 2010, p. 1-14.

-BIDART, DEGENNE, GROSSETTI, 2011 : Claire Bidart, Alain Degenne, Michel Grossetti, La Vie en réseau. Dynamiques des relations sociales, Paris, PUF, 2011.

- BOURDIEU, 1980 : Pierre Bourdieu, "Le capital social ", dans Actes de la recherche en sciences sociales, $\mathrm{n}^{\circ} 31$, janvier 1980, p. 2-3.

- Bourdieu, 1992 : Pierre Bourdieu, Les Règles de l'art. Genèse et structure du champ littéraire, Paris, Éditions du Seuil, 1992.

- BRESC-BAUtIER, 2017 : Geneviève Bresc-Bautier, "Les réseaux des sculpteurs parisiens sous Henri IV et Louis XIII", dans Jacques Verger (dir.), La Forme des réseaux : France et Europe ( $X^{e}-X X^{e}$ siècle), actes de colloque (Reims, 2015), Paris, CTHS, 2017, p. 9-25.

- BROSENS, KeLCHTERMANS, VAN DER STIGHELEN, 2012 : Koenraad Brosens, Leen Kelchtermans et Katlijne Van der Stighelen (dir.), Family Ties: Art Production and Kinship Patterns in the Early Modern Low Countries, Turnhout, Brepols 2012.

- CheVrefils-Desbiolles, Froissart-PeZONe, 2011 : Yves Chevrefils-Desbiolles, Rossella Froissart-Pezone (dir.), Les Revues d'art. Formes, stratégies et réseaux au $X X^{e}$ siècle, actes de colloque (Aix-en-Provence, 2008), Rennes, Presses universitaires de Rennes, 2011.

- COLEMAN, 1988 : James S. Coleman, "Social Capital in the Creation of Human Capital », dans The American
Journal of Sociology, no 94 : Organizations and Institutions: Sociological and Economic Approaches to the Analysis of Social Structure, 1988, p. S95-S120.

- CROSSLEY, MCANDREW, WIDDOP, 2015 : Nick Crossley, Siobhán McAndrew, Paul Widdop, Social Networks and Music Worlds, Abingdon / New York, Routledge, 2015.

- DACOS, MOUNIER, 2015 : Marin Dacos, Pierre Mounier, Humanités numériques. État des lieux et positionnement de la recherche française dans le contexte international, rapport ministériel, 2015 [en ligne, URL : www.enssib. fr/bibliotheque-numerique/notices/65358-humanitesnumeriques-etat-des-lieux-et-positionnement-de-larecherche-francaise-dans-le-contexte-international, consulté le 12 décembre 2017].

- DÉCULTOT, ESPAGNE, MARTIN, 2009 : Élisabeth Décultot, Michel Espagne, François-René Martin (dir.), Johann Georg Wille et son milieu. Un réseau européen de l'art au XVIII siècle, actes de colloque (Paris, 2007), Paris, École du Louvre, 2009.

- Dedieu, Chaparro, 2013 : Jean Pierre Dedieu, Álvaro Chaparro, «De la base au réseau. L'apport des bases de données à l'étude des réseaux ", dans Les Cahiers de Framespa. Nouveaux champs de l'histoire sociale, $\mathrm{n}^{\circ}$ 12, 2013 [en ligne, URL : journals.openedition.org/ framespa/2173, consulté le 12 novembre 2017].

- DEGENNE, 1978 : Alain Degenne, "La construction et l'analyse des réseaux sociaux ", dans L'Année sociologique, ${ }^{\circ} 29,1978$, p. 283-310.

- DeGenNe, Forsé, 2004 : Alain Degenne, Michel Forsé, Les Réseaux sociaux, Paris, Armand Colin, 2004.

\section{-}

- EVE, 2002 : Michael Eve, « Deux traditions d'analyse des réseaux sociaux ", dans Réseaux, nº 115, 2002-5, p. 183-212.

- FERRAND, FederICO, 2013 : Alexis Ferrand et Ainhoa de Federico, "L'analyse des réseaux sociaux en France: émergence (1977-1991) et diffusion des compétences (2005-2013) », dans Socio-logos, n 8, 2013 [en ligne, URL: socio-logos.revues.org/2794, consulté le 10 octobre 2017]. - FREEMAN, 2004 : Linton Freeman, The Development of Social Network Analysis: A Study in the Sociology of Science, Vancouver, Empirical Press, 2004.

- GADY, 2010 : Bénédicte Gady, L'Ascension de Charles Le Brun. Liens sociaux et production artistique, Paris, Éditions de la Maison des sciences de l'homme, 2010. - GranOVETTER, 1973 : Marc S. Granovetter, "The Strenght of Weak Ties ", dans American Journal of Sociology, no 78, mai 1973, p. 1360-1380.

- GrIBAUDI, 1998 : Maurizio Gribaudi (dir.), Espaces, Temporalités, Stratifications. Exercices sur les réseaux sociaux, Paris, Éditions de l'EHESS, 1998.

\section{-}

- HASKELL, (1963) 1991 : Francis Haskell, Mécènes et peintres. L'art et la société au temps du baroque italien, Fabienne Durand-Bogaert, Andrée Lyotard-May et Louis Evrard (trad. fra.), Paris, 1991 [éd. orig. : Patrons and Painters: A Study in the Relations between Italian Art and Society in the Age of the Baroque, New York, Knopf, 1963]. - HASSLER, 2015 : Éric Hassler, "Mettre en lumière des réseaux invisibles pour les historiens : la bipolarisation 
d'un réseau aristocratique à la cour de Vienne, 16851740 ", dans Les Cahiers de Framespa. Nouveaux champs de l'histoire sociale, no 18, 2015 [en ligne, URL : journals. openedition.org/framespa/3265, consulté le 24 novembre 2017].

- JOYEUX-PRUNEL, 2008 : Béatrice Joyeux-Prunel, « L'histoire de l'art et le quantitatif ", dans Histoire \& mesure, $n^{\circ}$ 23/2, 2008, p. 3-34 [en ligne, URL : histoiremesure. revues.org/3543, consulté le 5 décembre 2017].

- KARSALLAH et al., 2017 : Elsa Karsallah et al., « Introduction. Replacer la tapisserie au sein de l'histoire de la création artistique", dans Arachné. Un regard critique sur l'histoire de la tapisserie, Rennes, Presses universitaires de Rennes, 2017, p. 7-11 [en ligne, URL : www.pur-editions.fr/couvertures/1496407712_doc.pdf, consulté le 12 janvier 2018].

- KLAPISCH-ZUBER, 1990 : Christiane Klapisch-Zuber, La Maison et le Nom. Stratégies et rituels dans I'Italie de la Renaissance, Paris, Éditions de l'EHESS, 1990.

- KLAPISCH-ZUBER, 2003 : Christiane Klapisch-Zuber, L'Arbre des familles, Paris, La Martinière, 2003.

\section{-}

- LeMERCIER, 2005 : Claire Lemercier, "Analyse de réseaux et histoire ", dans Revue d'histoire moderne et contemporaine, no 52-2, avril-juin 2005, p. 88-112.

- MERCKLÉ, 2003-2004 : Pierre Mercklé, "Les réseaux sociaux. Les origines de l'analyse des réseaux sociaux ", CNED/ENS-ISH, 2003-2004.

- MERCKLÉ, 2004 : Pierre Mercklé, La Sociologie des réseaux sociaux, Paris, La Découverte, 2004.

- MONTIAS, 1982 : John Mickael Montias, Artists and Artisans in Delft: A Socio-economic Study of the Seventeenth Century, Princeton, Princeton University Press, 1982.

- MONTIAS, 1989 : John Mickael Montias, Vermeer and his Milieu: A Web of Social History, Princeton, Princeton University Press, 1989.

- MOULIN, 1967 : Raymonde Moulin, Le Marché de la peinture en France, Paris, Les Éditions de Minuit, 1967. - MOULIN, 1992 : Raymonde Moulin, L'Artiste, l'institution et le marché, Paris, Flammarion, 1992.

- NeRLICH, 2009 : France Nerlich (dir.), Interactions et transferts artistiques, numéro thématique de Histoire de l'art, nº 64, 2009.

- NERLICH, SAVOY, 2016 : France Nerlich, Bénédicte Savoy, "Projets croisés : ArtTransForm Regards croisés im Gespräch mit France Nerlich (Université FrançoisRabelais Tours) und Bénédicte Savoy (Technische Universität Berlin) ", dans Regards croisés, nº 5, 2016, p. $161-172$

- NOOY, 2003 : Wouter de Nooy, « Fields and Network Analysis: Correspondence Analysis and Social Network Analysis in the Frame-Work of Field Theory ", dans Poetics, no 31/5-6, octobre-décembre 2003, p. 305-327.

- PADGETT, 2001 : «Introduction to Marriage and Elite Structure in Renaissance Florence, 1282-1500 ", dans Redes. Revista hispana para el analisis de redes sociales, no 21/2, décembre 2001, p. 34-41.
- PAdGett, 2010 : John F. Padgett, « Open Elite? Social Mobility, Marriage, and Family in Florence, 1282-1494", dans Renaissance Quarterly, n 63, 2010, p. 357-411. - PAdgett, ANSEll, 1993 : John F. Padgett, Christopher K. Ansell, "Robust Action and the Rise of the Medici, 1400-1434 ", dans American Journal of Sociology, no 98/6, 1993, p. 1259-1319.

- Penalva-ICHer, ElOIRE, 2017 : Élise Penalva-Icher, Fabien Eloire, "Networking in France: Is there a French School of Social Network Analysis? », dans The American Sociologist, no 48/3-4, décembre 2017, p. 382-401.

- RIELLO, 2007 : Giorgio Riello, « La globalisation de I'Histoire globale : une question disputée », dans Revue $d^{\prime}$ histoire moderne et contemporaine, no 54-5, 2007-5, p. 30-31.

- ROCHE, (1978) 1989 : Daniel Roche, Le Siècle des Lumières en province. Académies et académiciens provinciaux, 1680-1789 (1978), Paris, Éditions de l'EHESS, 1989.

- SenMartin, BerTRAND, 2015 : Émilie Senmartin, Michel Bertrand (dir.), Les Cahiers de Framespa. Nouveaux champs de l'histoire sociale, $\mathrm{n}^{\circ} 18$ : Analyse historique et réseaux. Réflexion sur la représentation graphique, 2015.

\section{-}

- WHITE, (1965) 1991 : Harrison C. White, Cynthia A. White, La Carrière des peintres au XIXe siècle. Du système académique au marché des impressionnistes, Antoine Jaccottet (trad. fra.), Jean-Paul Bouillon (préface), Paris, Flammarion, 1991 [éd. orig. : Canvases and Careers: Institutional Change in the French Painting World, New York / Londres, J. Wiley and Sons, 1965]. - WHITE, 1992 : Harrison C. White, Identity and Control, Princeton, Princeton University Press, 1992.

- WHITE, 1993 : Harrison C. White, Careers and Creativity: Social Forces in the Arts, Boulder, Westview Press, 1993.

- Williams, 2015 : Hannah Williams, Académie Royale: A History in Portraits, Farnham, Ashgate, 2015. - WOOlLEtT, VAN SUCHTELEN, 2006 : Anne T. Woollett, Ariane Van Suchtelen (dir.), Rubens \& Brueghel: A Working Friendship, cat. exp. (Los Angeles, J. Paul Getty Museum ; La Haye, Royal Picture Gallery Mauritshuis, 2006-2007), Zwolle, Waanders, 2006. 


\section{WEBOGRAPHIE}

- Programme Les académies d'art et leurs réseaux dans la France préindustrielle (ACA-RES) : https://acares. hypotheses.org, consulté le 20 décembre 2017.

- Comité des travaux historiques et scientifiques, $140^{e}$ congrès, Reims, 2015, "Réseaux et société ": http://cths.fr/co/liste.php, consulté le 18 novembre 2017.

- Groupe Réseaux et Histoire (RES-HIST) : http://reshist. hypotheses.org, consulté le 20 novembre 2017.

- Labex Structurations des mondes sociaux (SMS) : http:// sms.univ-tlse2.fr/accueil-sms/le-labex-sms, consulté le 20 janvier 2018.

- GDR Analyse de réseaux en sciences humaines et sociales (AR-SHS) : https://arshs.hypotheses.org, consulté le 20 janvier 2018.

- Agence nationale de la recherche : http://www.agencenationale-recherche.fr/investissements-d-avenir, consulté le 22 décembre 2017.

- Programme Mapping the Republic of Letters : http:// republicofletters.stanford.edu, consulté le 3 mai 2017.

- Programme Van Gogh Letters : http://vangoghletters. org/vg, consulté le 23 novembre 2017.

- Laboratoire de recherche historique Rhône-Alpes LARHRA, UMR 5190, pôle d'histoire numérique : http:// larhra.ish-lyon.cnrs.fr/pole-histoire-numerique, consulté le 12 janvier 2018.

- Projet SymoGIH : http://symogih.org, consulté le 12 janvier 2018.

- Base ECARTICO, Linking cultural industries in the early modern Low Countries, ca. 1475-ca. 1725 : http:// www.vondel.humanities.uva.nl/ecartico, consulté le 11 février 2018.

- Programme Mapping Art Markets in Europe, 1500 1800 : http://www.artmarkets.eu/presentation, consulté le 2 février 2018.

- Édition numérique collaborative et critique de l'Encyclopédie (ENCREE) : http://enccre.academie-sciences. fr/encyclopedie, consulté le 12 janvier 2018.

- Programme Buffon et l'histoire naturelle : l'édition en ligne : http://www.buffon.cnrs.fr, consulté le 20 octobre 2017.

- Programme «Réseaux et sociabilité de cour », Centre de recherche du château de Versailles : https://www. chateauversailles-recherche.fr/francais/recherche/ programmes-de-recherche/programmes-de-rechercheen-cours/reseaux-et-sociabilite-a-la-cour, consulté le 11 février 2018.

- Unité mixte InVisu (CNRS/INHA) : https://www.inha. $\mathrm{fr} / \mathrm{fr} /$ recherche/l-unite-invisu-cnrs-inha.html, consulté le 11 février 2018.

- Conférence de Pierre-Michel Menger, Paris, Bibliothèque nationale de France, 16 mars 2017 : http://www. bnf.fr/fr/evenements_et_culture/anx_conferences_2017/ a.c_170316_petite_bibliotheque_sciences_sociales. html, consulté le 12 décembre 2017. 